\title{
Optimal Boundary Estimates for Stokes Systems in Homogenization Theory
}

\author{
$\mathrm{Shu} \mathrm{Gu}^{*}$ \\ Department of Mathematics, Florida State University, \\ Tallahassee, FL 32306-4510, USA. \\ Qiang $\mathrm{Xu}{ }^{\dagger}$ \\ School of Mathematical Sciences, Peking University, \\ Beijing, 100871, PR China.
}

August 7, 2018

\begin{abstract}
The paper concerns the sharp boundary regularity estimates in homogenization of Dirichlet problem for Stokes systems. We obtain the Lipschitz estimates for velocity term and $L^{\infty}$ estimate for pressure term, under some reasonable smoothness assumption on rapidly oscillating periodic coefficients. The approach is based on convergence rates, originally investigated by $\mathrm{S}$. Armstrong and Z. Shen in $[2,17]$, however the argument developed here does not rely on the Rellich estimates. In this sense, we find a new way to obtain the sharp uniform boundary estimates without imposing the symmetry assumption on coefficients. Additionally, we emphasize that $L^{\infty}$ estimate for the pressure term does require the $O\left(\varepsilon^{1 / 2}\right)$ convergence rate, locally at least, compared to $O\left(\varepsilon^{\lambda}\right)$ for the velocity term, where $\lambda \in(0,1 / 2)$.
\end{abstract}

Key words. Homogenization; Stokes system; Boundary estimates; Dirichlet problem.

\section{Instruction and main results}

In the paper [13], the first author and Z. Shen have systematically established the uniform estimates for Stokes systems with rapidly oscillating periodic coefficients, such as the $W^{1, p}$, Hölder estimates, and interior Lipschitz estimates. The compactness argument in [13], however, may not be directly applicable in obtaining the boundary Lipschitz estimate for the velocity yet, because of the lack of Green function estimates of Stokes systems with variable coefficients. In this paper, we would like to investigate the sharp boundary estimates of Dirichlet problem, by using the convergence rate estimates instead.

To be precise, we consider the following Stokes systems with the Dirichlet boundary condition

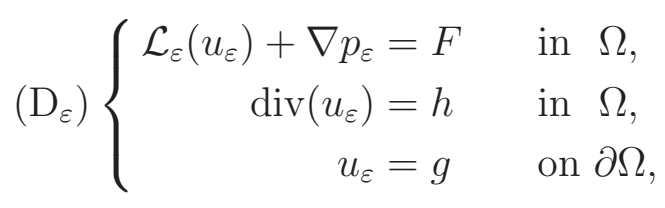

*Email: gu@math.fsu.edu.

†Email: xuqiang@math.pku.edu.cn. 
with the compatibility condition

$$
\int_{\Omega} h d x=\int_{\partial \Omega} n \cdot g d S
$$

where $n$ is the unit outward normal to $\partial \Omega, \varepsilon>0$ is a small parameter, and the operator $\mathcal{L}_{\varepsilon}$ is defined by

$$
\mathcal{L}_{\varepsilon}=-\operatorname{div}[A(x / \varepsilon) \nabla]=-\frac{\partial}{\partial x_{i}}\left[a_{i j}^{\alpha \beta}\left(\frac{x}{\varepsilon}\right) \frac{\partial}{\partial x_{j}}\right]
$$

Here $d \geq 2$ and $1 \leq i, j, \alpha, \beta \leq d$, and the summation convention for repeated indices is used throughout. We now assume that the coefficient matrix $A=\left(a_{i j}^{\alpha \beta}\right)$ is real and satisfies the uniform ellipticity condition

$$
\mu|\xi|^{2} \leq a_{i j}^{\alpha \beta}(y) \xi_{i}^{\alpha} \xi_{j}^{\beta} \leq \mu^{-1}|\xi|^{2} \quad \text { for } y \in \mathbb{R}^{d} \text { and } \xi=\left(\xi_{i}^{\alpha}\right) \in \mathbb{R}^{d \times d}, \quad \text { where } \mu>0 ;
$$

and the periodicity condition

$$
A(y+z)=A(y) \quad \text { for } y \in \mathbb{R}^{d} \text { and } z \in \mathbb{Z}^{d} .
$$

We also impose the smoothness condition, i.e.,

$$
|A(x)-A(y)| \leq \kappa|x-y|^{\tau} \quad \text { for } x, y \in \mathbb{R}^{d}, \quad \text { where } \tau \in(0,1) .
$$

The following is the main result of the paper.

Theorem 1.1. Let $\Omega$ be a bounded $C^{1, \tau}$ domain. Suppose A satisfies (1.2) - (1.4). Given $F \in$ $L^{p}\left(\Omega ; \mathbb{R}^{d}\right)$ and $h \in W^{1, p}(\Omega)$ with $p>d$, and $g \in C^{1, \eta}\left(\Omega ; \mathbb{R}^{d}\right)$ satisfying the compatibility condition (1.1), where $\eta \in(0, \tau]$, let $\left(u_{\varepsilon}, p_{\varepsilon}\right) \in H^{1}\left(\Omega ; \mathbb{R}^{d}\right) \times L^{2}(\Omega)$ be a weak solution of the Stokes system $\left(\mathrm{D}_{\varepsilon}\right)$. Then we have the uniform estimate

$$
\left\|\nabla u_{\varepsilon}\right\|_{L^{\infty}(\Omega)}+\left\|p_{\varepsilon}-f_{\Omega} p_{\varepsilon}\right\|_{L^{\infty}(\Omega)} \leq C\left\{\|F\|_{L^{p}(\Omega)}+\|h\|_{W^{1, p}(\Omega)}+\|g\|_{C^{1, \eta}(\partial \Omega)}\right\},
$$

where $C$ depends only on $\mu, \kappa, \tau, d, \eta, p$ and $\Omega$.

We mention that the estimate (1.5) is sharp even with the $C^{\infty}$ data and domains. Let us first recap the important development in quantitative homogenization theory, especially in the periodic settings. In the late 1980s, uniform regularity estimates for elliptic systems with Dirichlet boundary conditions was first proved by M. Avellandeda and F. Lin [3], where the compactness method was introduced. However, it was not until 2013 that the regularity estimates for the elliptic Neumann boundary problems was solved by C. Kenig, F. Lin and Z. Shen [14]. Another recent breakthrough was made by S. Armstrong and Z. Shen in [2] for the almost-periodic setting, and they developed a new method which based on convergence rates rather than the compactness methods. We refer the reader to $[1,19,26]$ and its reference therein for more details on non-periodic cases. Meanwhile, T. Suslina $[20,21]$ obtained the sharp $O(\varepsilon)$ convergence rates in $L^{2}(\Omega)$ for elliptic homogenization problems in $C^{1,1}$ domains, while C. Kenig, F. Lin, and Z. Shen [15] figured out the almost sharp one $O(\varepsilon \ln (1 / \varepsilon))$ concerned with Lipschitz domains, and their results have been improved by the second author in [23], recently. If the reader interests in the boundary estimates, we highly recommend Z. Shen's elegant work [17]. The quantitative homogenization has been extensively studied, we refer the reader to $[1,2,3,4,7,8,9,10,13,18,19,24,25]$ and their references therein.

For the case of Stokes systems $\left(D_{\varepsilon}\right)$, the uniform interior estimates and boundary Hölder estimates for the Dirichlet problem have already been established by the first author and Z. Shen [13]. Now we 
only focus ourselves on the corresponding boundary estimates. For simplicity, we use the following notation throughout. Let

$$
\begin{aligned}
& D_{r}=\left\{\left(x^{\prime}, x_{d}\right) \in \mathbb{R}^{d}:\left|x^{\prime}\right|<r \text { and } \psi\left(x^{\prime}\right)<x_{d}<\psi\left(x^{\prime}\right)+r\right\}, \\
& \Delta_{r}=\left\{\left(x^{\prime}, x_{d}\right) \in \mathbb{R}^{d}:\left|x^{\prime}\right|<r \text { and } x_{d}=\psi\left(x^{\prime}\right)\right\},
\end{aligned}
$$

where $\psi: \mathbb{R}^{d-1} \rightarrow \mathbb{R}$ is a $C^{1, \tau}$ function for some $\tau \in(0,1)$ with $\psi(0)=0$ and $\|\nabla \psi\|_{C^{0, \tau}\left(\mathbb{R}^{d-1}\right)} \leq M$.

Theorem 1.2 (Local boundary estimates). Assume the same conditions as in Theorem 1.1. Let $\left(u_{\varepsilon}, p_{\varepsilon}\right) \in H^{1}\left(D_{5} ; \mathbb{R}^{d}\right) \times L^{2}\left(D_{5}\right)$ be a weak solution of $\mathcal{L}_{\varepsilon}\left(u_{\varepsilon}\right)+\nabla p_{\varepsilon}=F$ and $\operatorname{div}\left(u_{\varepsilon}\right)=h$ in $D_{5}$ and $u_{\varepsilon}=g$ on $\Delta_{5}$, where $F \in L^{p}\left(D_{5} ; \mathbb{R}^{d}\right)$ and $h \in W^{1, p}\left(D_{5}\right)$ with $p>d$, and $g \in C^{1, \eta}\left(\Delta_{5} ; \mathbb{R}^{d}\right)$ with $g(0)=0$, where $\eta \in(0, \tau]$. Then there holds

$$
\begin{aligned}
\left(f_{D_{r}}\left|\nabla u_{\varepsilon}\right|^{2} d x\right)^{\frac{1}{2}}+\left|f_{D_{r}} p_{\varepsilon}-f_{D_{1}} p_{\varepsilon} d x\right| \leq C & \left\{\left(f_{D_{2}}\left|u_{\varepsilon}\right|^{2} d x\right)^{\frac{1}{2}}+\left(f_{D_{2}}|F|^{p} d x\right)^{\frac{1}{p}}\right. \\
+ & \left.\left(f_{D_{2}}|\nabla h|^{p} d x\right)^{\frac{1}{p}}+\|h\|_{L^{\infty}\left(D_{2}\right)}+\|g\|_{C^{1, \eta}\left(\Delta_{2}\right)}\right\}
\end{aligned}
$$

for any $0<r<1 / 4$, where $C$ depends only on $\mu, \kappa, \tau, d, M, \eta$ and $p$.

The scaling-invariant estimate (1.6) ought to be regarded as a Lipschitz estimate for the velocity $u_{\varepsilon}$ and $L^{\infty}$ estimate for the pressure $p_{\varepsilon}$, since it is not hard to bound the quantity

$$
\left|\nabla u_{\varepsilon}(0)\right|+\left|p_{\varepsilon}(0)-f_{D_{1}} p_{\varepsilon}\right|
$$

by the right-hand side of (1.6) due to the blow-up argument, where $0 \in \partial \Omega$. Before explaining our tactics, let us review the ideas developed in $[2,17]$. For elliptic operator $\mathcal{L}_{\varepsilon}$ with Dirichlet or Neumann boundary conditions, they found that the quantity

$$
\left(f_{D_{r}}\left|\nabla u_{\varepsilon}\right|^{2} d x\right)^{1 / 2}
$$

could be bounded for any scale $r$ uniformly down to $\varepsilon$, provided the coefficients own some repeated self-similar structure, for example, which may be periodic or almost-periodic, even random in stationary and ergodic setting. This result indeed came from the so-called Campanato iteration. However, it requires an effective control of the error in homogenization as a precondition. Take the periodic homogenization as an example, Z. Shen [17] construct a function $v$ such that $\mathcal{L}_{0}(v)=F$ in $D_{r}$ with the same (Dirichlet or Neumann) data on $\Delta_{r}$ as $u_{\varepsilon}$, where $\mathcal{L}_{0}$ is the homogenized (effective) operator of $\mathcal{L}_{\varepsilon}$, and

$$
\left(f_{D_{r}}\left|u_{\varepsilon}-v\right|^{2} d x\right)^{1 / 2} \leq C\left(\frac{\varepsilon}{r}\right)^{1 / 2}\left\{\left(f_{D_{2 r}}\left|u_{\varepsilon}\right|^{2} d x\right)^{\frac{1}{2}}+\text { terms involving given data }\right\} .
$$

It is a scaling-invariant estimate, so it suffices to consider the case of $r=1$, and this is exactly where the convergence rates $\left\|\mathcal{L}_{\varepsilon}^{-1}-\mathcal{L}_{0}^{-1}\right\|_{L^{2}(\Omega) \rightarrow L^{2}(\Omega)}=O\left(\varepsilon^{1 / 2}\right)$ play a role. We mention that this estimate is not as simple as it appears, even for elliptic systems in a bounded $C^{1, \tau}$ domain. The hard part is to control the second order derivative lacking the smoothness assumptions on coefficients or domains. However, if the operator $\mathcal{L}_{\varepsilon}$ satisfies a symmetry condition, i.e., $A=A^{*}$, then the Rellich estimate 
became a powerful tool, which makes it possible to use the nontangential maximal function to control the boundary behavior of the solution. We mention that the estimate (1.7) is established in Lipschitz domains without any smoothness assumption on coefficients, but it relies on the symmetry condition. We refer the reader to $[2,17]$ for the original thinking.

In the paper, although the main idea is similar as that in $[2,17]$, the innovation clearly reflects in two aspects: the estimate (1.6) does not depend on any symmetry condition, which can be extended to the Neumann boundary problems without real difficulties; using convergence rates on pressure term recovers its uniform boundary $L^{\infty}$ estimate, which shows an approach unlike the iteration arguments applied to the velocity term.

In the following paragraphs, we will outline our strategy related to the estimate (1.6). As the second author has found in [23], originally motivated by Z. Shen in [17], the order of the convergence rates is determined by the so-called "layer" and "co-layer" type estimates for the homogenized boundary problems. If $\Omega$ is a bounded $C^{1}$ domain, the interior Schauder estimate combining with the global Hölder estimate leads to

$$
\left\|u_{\varepsilon}-u_{0}\right\|_{L^{2}(\Omega)} \leq C \varepsilon^{\sigma-\frac{1}{2}}\left\{\|F\|_{L^{p}(\Omega)}+\|h\|_{W^{1, p}(\Omega)}+\|g\|_{C^{0,1}(\partial \Omega)}\right\}
$$

for any $\sigma \in(1 / 2,1)$. Hence, under the same assumptions as in Theorem 1.2, we construct the solution $\left(u_{0}, p_{0}\right)$ satisfying $\mathcal{L}_{0}\left(u_{0}\right)+\nabla p_{0}=F$, and $\operatorname{div}\left(u_{0}\right)=h$ in $D_{2}$, and $u_{0}=u_{\varepsilon}$ on $\partial D_{2}$, it follows from the estimate (1.8) that

$$
\left\|u_{\varepsilon}-u_{0}\right\|_{L^{2}\left(D_{1}\right)} \leq C \varepsilon^{\sigma-\frac{1}{2}}\left\{\left\|\nabla u_{\varepsilon}\right\|_{L^{\infty}\left(\partial D_{2} \backslash \Delta_{2}\right)}+\text { terms involve given data }\right\} .
$$

Obviously, the challenging task is to estimate the quantity $\left\|\nabla u_{\varepsilon}\right\|_{L^{\infty}\left(\partial D_{2} \backslash \Delta_{2}\right)}$. Although we can not count on bounding it uniformly, it is possible to derive a nonuniform estimate for the first term of the right-hand side of (1.9), and we hope that its "diverging order" will be smaller than $\sigma-\frac{1}{2}$. In fact, the local Lipschitz estimate together with the uniform global Hölder estimate gives us

$$
\left\|\nabla u_{\varepsilon}\right\|_{L^{\infty}\left(\partial D_{2} \backslash \Delta_{2}\right)} \leq C \varepsilon^{\sigma-1}\left\{\left\|u_{\varepsilon}\right\|_{L^{2}\left(D_{4}\right)}+\text { terms involve given data }\right\} .
$$

Combining the estimates (1.9) and (1.10), we arrive at

$$
\left\|u_{\varepsilon}-u_{0}\right\|_{L^{2}\left(D_{1}\right)} \leq C \varepsilon^{\lambda}\left\{\left\|u_{\varepsilon}\right\|_{L^{2}\left(D_{4}\right)}+\text { terms involve given data }\right\},
$$

where $\lambda=2 \sigma-\frac{3}{2}$. It is clear to see that $\lambda \in(0,1 / 2)$ whenever $(3 / 4)<\sigma<1$. We emphasize that we just require a positive power $\lambda$ to proceed the Campanato iteration for Lipschitz estimates on velocity $u_{\varepsilon}$.

Compared to the methods developed in [17], ours involve with the microcosmic information on coefficients, which may be regarded as the price of sacrificing the symmetry conditions. The lucky thing is that the requirements of additional smoothness assumptions are not beyond those in common cases. Another remark is that we essentially use the uniform Hölder estimates in (1.10), which have already been established in [13] by the well known compactness method. In this sense, the arguments developed here actually blend the compactness methods, convergence rates coupled with the Campanato iteration. Here the iteration argument (see Lemma 4.6) belongs to Z. Shen, who notably simplified the proof in [17]. If the model is replaced by elliptic systems, we may even obtain sharp Hölder estimates for Dirichlet problems, that means it suffices to bound the quantity $\left\|u_{\varepsilon}\right\|_{C^{0, \eta^{\prime}}\left(\partial D_{2} \backslash \Delta_{2}\right)}$, where $\eta^{\prime} \in(0,1)$, and it will be done by the uniform Hölder estimates without the 
blow-up arguments. However, the case of Neumann boundary follows the same way as we addressed here, since one has to estimate the quantity $\left\|\nabla u_{\varepsilon}\right\|_{L^{\infty}\left(\partial D_{2} \backslash \Delta_{2}\right)}$, as well. To some extend, this suggests that the Neumann problem will be more complicated than the Dirichlet one. We will show the Stokes systems with the Neumann boundary conditions in a separate work. The paragraph ends here by mentioning that the idea of a nonuniform estimate has already been used in the study of elliptic systems with lower order terms by the second author (see [24]).

We now turn to show how to bound the pressure $p_{\varepsilon}$ in $L^{\infty}$-norm, which reflects the other innovation of the paper. Note that Lipschitz estimates for $u_{\varepsilon}$ do not simply implies the $L^{\infty}$ estimate for $p_{\varepsilon}$, since $p_{\varepsilon}$ is related to $\nabla u_{\varepsilon}$ by a singular integral. We find that due to the local Schauder estimates for $\mathcal{L}_{0}$, it is not hard to derive

$$
\left(f_{D_{r}}\left|p_{0}-f_{D_{s}} p_{0}\right|^{2} d x\right)^{\frac{1}{2}} \leq C s^{\rho}\left\{\left\|u_{0}\right\|_{L^{2}\left(D_{2}\right)}+\text { terms involve given data }\right\}
$$

for any $0<r \leq s \leq 1$, where $0<\rho<\min \{\eta, 1-d / p\}$. In this form, the above estimate is quite similar to the desired estimate for $p_{\varepsilon}$. Hence, roughly speaking, the idea is to transfer the corresponding estimate for $p_{\varepsilon}$ to a similar one for $p_{0}$ by using the convergent relationship between $p_{\varepsilon}$ and $p_{0}$. However, the first intractable problem is that $p_{\varepsilon}$ just weakly converges to $p_{0}$ in $L^{2}(\Omega)$, and we can not expect any precise control of the error. Fortunately, it is known that $p_{\varepsilon}$ subtracting its first order approximating corrector strongly converges in $L^{2}(\Omega) / \mathbb{R}$, where $L^{2}(\Omega) / \mathbb{R}$ denotes the quotient space of $L^{2}(\Omega)$ with respect to the relation $u \sim v \Leftrightarrow u-v \in \mathbb{R}$. As will be shown in Section 5 , we indeed obtain

$$
\begin{aligned}
& \| p_{\varepsilon}-p_{0}-\pi(\cdot / \varepsilon) \psi_{2 \varepsilon} \nabla u_{0} \|_{L^{2}\left(D_{1}\right) / \mathbb{R}} \\
& \leq C \varepsilon^{\lambda}\left\{\left\|u_{\varepsilon}\right\|_{L^{2}\left(D_{4}\right)}+\text { terms involve given data }\right\}
\end{aligned}
$$

where $(\chi, \pi)$ is the corrector associated with $\left(\mathrm{D}_{\varepsilon}\right)$, defined in Subsection 2.3 , and $\psi_{2 \varepsilon}$ is a smooth cutoff function whose expression will be given in Subsection 2.1. Although the estimate (1.12) provides us an accurate way to control the rate of the convergence, the control, in fact, is required in each scale $r$ from $\varepsilon$ to 1 . That means, for example, to bound the quantity $\left|f_{D_{r}} p_{\varepsilon}-f_{D_{1}} p_{\varepsilon} d x\right|$ for any $\varepsilon \leq r<(1 / 4)$, it suffices to consider the quantity

$$
\sum_{j=1}^{k}\left|f_{D_{2^{-j}}} p_{\varepsilon}-f_{D_{2^{-j+1}}} p_{\varepsilon} d x\right|
$$

where $2^{-k-1} \leq r<2^{-k}$. Hence we need the scaling-invariance version of (1.12) and it will be established in Lemma 5.2. Besides, in the calculation, we also need to estimate the quantity

$$
\frac{1}{\left|D_{2^{-j}}\right|^{\frac{1}{2}}}\left\{\left\|\nabla u_{0}\right\|_{L^{2}\left(D_{2^{-j}} \backslash \Sigma_{4 \varepsilon}\right)}+\varepsilon\left\|\nabla^{2} u_{0}\right\|_{L^{2}\left(D_{2^{-j}} \cap \Sigma_{4 \varepsilon}\right)}\right\}
$$

where $\Sigma_{4 \varepsilon}=\{x \in \Omega: \operatorname{dist}(x, \partial \Omega)>4 \varepsilon\}$, and it will be controlled by

$$
C\left(\varepsilon 2^{j}\right)^{\frac{1}{2}}\left\{\left\|u_{0}\right\|_{L^{2}\left(D_{2}\right)}+\text { terms involve given data }\right\} .
$$

The concrete statement can be found in Lemma 3.4. The above expression also reveals that the convergence rate related to pressure term must reach $O\left(\varepsilon^{1 / 2}\right)$ in the local sense, and it guarantees 
that $\sum_{j=1}^{k}\left(\varepsilon 2^{j}\right)^{\frac{1}{2}}$ is convergent whenever $0<\varepsilon<2^{-k}$. Compared to $O\left(\varepsilon^{\lambda}\right)$ rates for the velocity term, where $\lambda \in(0,1 / 2)$, it seems to be an evidence that the $L^{\infty}$ estimate for the pressure term is harder than the Lipschitz estimate for the velocity term. In the last step of the proof, we use the fact that $u_{\varepsilon}$ strongly converges to $u_{0}$ in $L^{2}\left(D_{2}\right)$ due to the homogenization theory. We finally remark that the case of $0<r \leq \varepsilon$ always follows from the blow-up argument.

The paper is organized as follows. Section 2 is divided into four subsections, including notation, smoothing operator and its properties, corrector and its properties, and the classical regularity theory. Section 3 is devoted to study the rate of convergence. We show the uniform Lipschitz estimates on velocity $u_{\varepsilon}$ in Section 4 , and the $L^{\infty}$ estimates on pressure $p_{\varepsilon}$ in Section 5.

\section{Preliminaries}

\subsection{Notation in the paper}

We first introduce some notation that will be used in the following sections.

- $\nabla v=\left(\nabla_{1} v, \cdots, \nabla_{d} v\right)$ is the gradient of $v$, where $\nabla_{i} v=\partial v / \partial x_{i}$ denotes the $i^{\text {th }}$ derivative of $v$. $\nabla^{2} v=\left(\nabla_{i j}^{2} v\right)_{d \times d}$ denotes the Hessian matrix of $v$, where $\nabla_{i j}^{2} v=\frac{\partial^{2} v}{\partial x_{i} \partial x_{j}}$. $\operatorname{div}(\mathbf{v})=\sum_{i=1}^{d} \nabla_{i} v_{i}$ denotes the divergence of $\mathbf{v}$, where $\mathbf{v}=\left(v_{1}, \cdots, v_{d}\right)$ is a vector-valued function.

- $L^{2}(\Omega) / \mathbb{R}=\left\{f \in L^{2}(\Omega): \int_{\Omega} f(x) d x=0\right\}$, and $\|f\|_{L^{2}(\Omega) / \mathbb{R}}=\inf _{c \in \mathbb{R}}\|f-c\|_{L^{2}(\Omega)}$.

- $\delta(x)=\operatorname{dist}(x, \partial \Omega)$ denotes the distance function for $x \in \Omega$, and we set $\delta(x)=0$ if $x \in \mathbb{R}^{d} \backslash \Omega$.

- $S_{r}=\{x \in \Omega: \operatorname{dist}(x, \partial \Omega)=r\}$ denotes the level set.

- $\Omega \backslash \Sigma_{r}$ denotes the boundary layer with thickness $r>0$, where $\Sigma_{r}=\{x \in \Omega$ : $\operatorname{dist}(x, \partial \Omega)>r\}$.

- Let $B=B(x, r)=B_{r}(x)$, and $k B=B(x, k r)$ denote the concentric balls as $k>0$ varies.

- Let $\psi_{r}$ denote the cut-off function associated with $\Sigma_{r}$, such that

$$
\psi_{r}=1 \quad \text { in } \Sigma_{2 r}, \quad \psi_{r}=0 \quad \text { outside } \Sigma_{r}, \quad \text { and } \quad\left|\nabla \psi_{r}\right| \leq C / r \text {. }
$$

Throughout the paper, the constant $C$ never depends on $\varepsilon$. Finally we mention that we shall make a little effort to distinguish vector-valued functions or function spaces from their real-valued counterparts, and they will be clear from the context.

\subsection{Smoothing operator and its properties}

We first state the definition and properties of the smoothing operator $S_{\varepsilon}$. Detailed proof may be found in [17]. We fix $\zeta \in C_{0}^{\infty}(B(0,1 / 2))$, and $\int_{\mathbb{R}^{d}} \zeta(x) d x=1$ and denote $\zeta_{\varepsilon}$ by $\zeta_{\varepsilon}=\varepsilon^{-d} \zeta(x / \varepsilon)$. The smoothing operator $S_{\varepsilon}$ is defined by

$$
S_{\varepsilon}(f)(x)=f * \zeta_{\varepsilon}(x)=\int_{\mathbb{R}^{d}} f(x-y) \zeta_{\varepsilon}(y) d y,
$$


Lemma 2.1. Let $f \in L^{p}\left(\mathbb{R}^{d}\right)$ for some $1 \leq p<\infty$. Then for any $\rho \in L_{\text {per }}^{p}\left(\mathbb{R}^{d}\right)$,

$$
\left\|\rho(\cdot / \varepsilon) S_{\varepsilon}(f)\right\|_{L^{p}\left(\mathbb{R}^{d}\right)} \leq C\|\rho\|_{L^{p}(Y)}\|f\|_{L^{p}\left(\mathbb{R}^{d}\right)},
$$

where $C$ depends only on $d$.

Lemma 2.2. Let $f \in W^{1, p}\left(\mathbb{R}^{d}\right)$ for some $1<p<\infty$. Then we have

$$
\left\|S_{\varepsilon}(f)-f\right\|_{L^{p}\left(\mathbb{R}^{d}\right)} \leq C \varepsilon\|\nabla f\|_{L^{p}\left(\mathbb{R}^{d}\right)},
$$

and further obtain

$$
\left\|S_{\varepsilon}(f)\right\|_{L^{2}\left(\mathbb{R}^{d}\right)} \leq C \varepsilon^{-1 / 2}\|f\|_{L^{q}\left(\mathbb{R}^{d}\right)} \quad \text { and } \quad\left\|S_{\varepsilon}(f)-f\right\|_{L^{2}\left(\mathbb{R}^{d}\right)} \leq C \varepsilon^{1 / 2}\|\nabla f\|_{L^{q}\left(\mathbb{R}^{d}\right)},
$$

where $q=2 d /(d+1)$, and $C$ depends only on $d$.

\subsection{Correctors and its properties}

We will use this subsection to introduce the correctors and dual correctors of Stokes systems in the homogenization theory. Details can be found in the literatures such as [13, 10, 11, 12, 22].

Let $Y=[0,1)^{d} \simeq \mathbb{R}^{d} / \mathbb{Z}^{d}$. We define the correctors $\left(\chi_{k}^{\beta \gamma}, \pi_{k}^{\gamma}\right) \in H_{p e r}^{1}\left(Y ; \mathbb{R}^{d}\right) \times L_{p e r}^{2}(Y)$ associated with the Stokes system $\left(D_{\varepsilon}\right)$ by the following cell problem:

$$
\left\{\begin{array}{rlrl}
\mathcal{L}_{1}\left(\chi_{k}^{\gamma}+P_{k}^{\gamma}\right)+\nabla \pi_{k}^{\gamma}=0 & & \text { in } \mathbb{R}^{d}, \\
\operatorname{div}\left(\chi_{k}^{\gamma}\right)=0 & \text { in } \mathbb{R}^{d}, \\
\int_{Y} \chi_{k}^{\gamma} d y=0, & & k, \gamma=1, \cdots, d,
\end{array}\right.
$$

where $P_{k}^{\gamma}=y_{k} e^{\gamma}=y_{k}(0, \cdots, 1, \cdots, 0)$ with 1 in the $\gamma^{\text {th }}$ position, and $\chi_{k}^{\gamma}=\left(\chi_{k}^{1 \gamma}, \cdots, \chi_{k}^{d \gamma}\right)$. It follows from [13, Theorem 2.1] that

$$
\left\|\nabla \chi_{k}^{\gamma}\right\|_{L^{2}(Y)}+\left\|\pi_{k}^{\gamma}\right\|_{L^{2}(Y)} \leq C
$$

where $C$ depends only on $\mu$ and $d$. Then the homogenized operator is given by $\mathcal{L}_{0}=-\operatorname{div}(\widehat{A} \nabla)$, where $\widehat{A}=\left(\hat{a}_{i j}^{\alpha \beta}\right)$ and

$$
\hat{a}_{i j}^{\alpha \beta}=\int_{Y}\left[a_{i j}^{\alpha \beta}+a_{i k}^{\alpha \gamma} \frac{\partial}{\partial y_{k}}\left(\chi_{j}^{\gamma \beta}\right)\right] d y
$$

By the homogenization theory proved in $[13,4]$, we know that

$$
u_{\varepsilon} \rightarrow u_{0} \text { weakly in } H^{1}\left(\Omega ; \mathbb{R}^{d}\right) \text { and } \quad p_{\varepsilon}-f_{\Omega} p_{\varepsilon} \rightarrow p_{0}-f_{\Omega} p_{0} \text { weakly in } L^{2}(\Omega),
$$

where $\left(u_{0}, p_{0}\right)$ is the weak solution of the homogenized problem,

$$
\left(\mathrm{D}_{0}\right)\left\{\begin{aligned}
\mathcal{L}_{0}\left(u_{0}\right)+\nabla p_{0}=F & \text { in } \Omega, \\
\operatorname{div}\left(u_{0}\right)=h & \text { in } \Omega, \\
u_{0}=g & \text { on } \partial \Omega .
\end{aligned}\right.
$$

We define

$$
b_{i k}^{\alpha \gamma}(y)=\hat{a}_{i k}^{\alpha \gamma}-a_{i k}^{\alpha \gamma}(y)-a_{i j}^{\alpha \beta}(y) \frac{\partial \chi_{k}^{\beta \gamma}}{\partial y_{j}}(y), \quad y=\frac{x}{\varepsilon},
$$

and it is obvious that $\int_{Y} b_{i k}^{\alpha \gamma} d y=0$, and $\nabla_{i} b_{i k}^{\alpha \gamma}=-\nabla_{\alpha} \pi_{k}^{\gamma}$. As usual, the following lemma provides the definition and the properties of $\left(E_{j i k}^{\alpha \gamma}, q_{i k}^{\gamma}\right)$ of Stokes systems, we refer [10, 11, 12, 22] for more details. 
Lemma 2.3. There exist $E_{j i k}^{\alpha \gamma} \in H_{p e r}^{1}(Y)$ and $q_{i k}^{\gamma} \in H_{p e r}^{1}(Y)$ such that

$$
b_{i k}^{\alpha \gamma}=\nabla_{j} E_{j i k}^{\alpha \gamma}-\nabla_{\alpha} q_{i k}^{\gamma}, \quad E_{j i k}^{\alpha \gamma}=-E_{i j k}^{\alpha \gamma}, \quad \text { and } \quad \nabla_{i} q_{i k}^{\gamma}=\pi_{k}^{\gamma},
$$

and $E_{j i k}^{\alpha \gamma}$ and $q_{i k}^{\gamma}$ admit the priori estimate

$$
\left\|E_{j i k}^{\alpha \gamma}\right\|_{L^{2}(Y)}+\left\|q_{i k}^{\gamma}\right\|_{L^{2}(Y)} \leq C
$$

where $C$ depends only on $\mu$ and $d$.

Lemma 2.4. Let $\left\{E_{j i k}^{\alpha \gamma}, q_{i k}^{\gamma}\right\}$ be given in Lemma 2.3. Additionally, if the correctors $\chi_{k}=\left(\chi_{k}^{\beta \gamma}\right)$ with $k=1, \cdots, d$ are Hölder continuous, then $E_{j i k}^{\alpha \gamma}, q_{i k}^{\gamma} \in L^{\infty}(Y)$.

Proof. See [22, Lemma 5.1]

Remark 2.5. If $\pi \in L_{\text {per }}^{2}(Y)$, there exists $V \in H_{\text {per }}^{1}\left(Y ; \mathbb{R}^{d}\right)$ such that $\operatorname{div}_{y}(V)=\pi(y)-\widehat{\pi}$ in $\mathbb{R}^{d}$. Moreover if $\pi \in L^{\infty}\left(\mathbb{R}^{d}\right)$, then we have $\|V\|_{L^{\infty}\left(\mathbb{R}^{d}\right)} \leq C(\mu, \tau, \kappa, d)$. The proof is similar to that in [22, Lemma 5.1].

\subsection{Classical regularity theory}

In this section, we recall some classical results, including the local and global versions, which mainly come from $[5,6,16]$ and the reference therein. To avoid confusion, we use the notation $\mathcal{L}(u)=\operatorname{div}[A(x) \nabla u]$ to denote the operator with variable coefficients that does not depend on $\varepsilon$.

Theorem 2.6 (Global Hölder estimates). Let $\Omega$ be a bounded $C^{1}$ domain. If $A \in \mathrm{VMO}$ satisfies (1.2). Given $F \in L^{p}\left(\Omega ; \mathbb{R}^{d}\right), h \in L^{2 p}(\Omega)$ with $2 p>d$ and $g \in C^{0,1}\left(\partial \Omega ; \mathbb{R}^{d}\right)$ satisfying the compatibility condition $(1.1)$, let $(u, \varrho) \in H^{1}\left(\Omega ; \mathbb{R}^{d}\right) \times L^{2}(\Omega)$ be the weak solution to the Dirichlet problem: $\mathcal{L}(u)+$ $\nabla \varrho=F$ and $\operatorname{div}(u)=h$ in $\Omega$ and $u=g$ on $\partial \Omega$. Then for any $0<\sigma<1$, we have

$$
\|u\|_{C^{0, \sigma}(\Omega)} \leq C\left\{\|F\|_{L^{p}(\Omega)}+\|h\|_{L^{2 p}(\Omega)}+\|g\|_{C^{0,1}(\partial \Omega)}\right\}
$$

where $C$ depends on $\mu, \omega, d, p, \sigma$ and $\Omega$.

Theorem 2.7 (Local estimates). Let $\Omega$ be a $C^{1, \tau}$ domain. Assume that A satisfies (1.2) and (1.4). Let $(u, \varrho) \in H^{1}\left(D_{5} ; \mathbb{R}^{d}\right) \times L^{2}\left(D_{5}\right)$ be the weak solution to $\mathcal{L}(u)+\nabla \varrho=F$ and $\operatorname{div}(u)=h$ in $D_{5}$, and $u=g$ on $\Delta_{5}$, where $F \in L^{p}\left(D_{5} ; \mathbb{R}^{d}\right)$ and $h \in W^{1, p}\left(D_{5}\right)$ with $p>d$, and $g \in C^{1, \eta}\left(\Delta_{5} ; \mathbb{R}^{d}\right)$ with $g(0)=0$. Then for any $0<\rho \leq \min \{\eta, 1-d / p\}$, we have the following estimate:

(1) Interior Schauder estimate, for any $B_{2 r} \subset D_{5}$, there holds

$$
\begin{aligned}
{[\nabla u]_{C^{0, \rho}\left(B_{r}\right)}+[\varrho]_{C^{0, \rho}\left(B_{r}\right)} } & \leq C r^{-\rho}\left\{\frac{1}{r}\left(f_{B_{2 r}}|u-c|^{2} d x\right)^{\frac{1}{2}}\right. \\
& \left.+r\left(f_{B_{2 r}}|F|^{p} d x\right)^{\frac{1}{p}}+r\left(f_{B_{2 r}}|\nabla h|^{p} d x\right)^{\frac{1}{p}}+\|h\|_{L^{\infty}\left(B_{2 r}\right)}\right\}
\end{aligned}
$$

for any $c \in \mathbb{R}^{d}$, where $C$ depends only on $\mu, \tau, \kappa, d, p$ and $\rho$. 
(2) Boundary Schauder estimate, for any $D_{2 r} \subset D_{5}$ and $c \in \mathbb{R}^{d}$, we have

$$
\begin{aligned}
{[\nabla u]_{C^{0, \rho}\left(D_{r}\right)}+[\varrho]_{C^{0, \rho}\left(D_{r}\right)} \leq } & C r^{-\rho}\left\{\frac{1}{r}\left(f_{D_{2 r}}|u|^{2} d x\right)^{\frac{1}{2}}+r\left(f_{D_{2 r}}|F|^{p} d x\right)^{\frac{1}{p}}+r\left(f_{D_{2 r}}|\nabla h|^{p} d x\right)^{\frac{1}{p}}\right. \\
& \left.+\|h\|_{L^{\infty}\left(D_{2 r}\right)}+\|\nabla g\|_{L^{\infty}\left(D_{2 r}\right)}+r^{\eta}[\nabla g]_{C^{0, \eta}\left(\Delta_{2 r}\right)}\right\}
\end{aligned}
$$

where $C$ depends on $\mu, \tau, \kappa, d, p, \eta, M$ and $\rho$.

(3) Boundary Lipschitz estimate, for any $0<r \leq R \leq 1$, we have

$$
\left.\begin{array}{rl}
\|\nabla u\|_{L^{\infty}\left(D_{r}\right)}+\| \varrho- & f_{D_{R}} \varrho \|_{L^{\infty}\left(D_{r}\right)} \leq C\left\{\frac{1}{R}\left(f_{D_{2 R}}|u|^{2} d x\right)^{\frac{1}{2}}+R\left(f_{D_{2 R}}|F|^{p} d x\right)^{\frac{1}{p}}\right. \\
& +R\left(f_{D_{2 R}}|\nabla h|^{p} d x\right)^{\frac{1}{p}}+\|h\|_{L^{\infty}\left(D_{2 R}\right)}+\|\nabla g\|_{L^{\infty}\left(D_{2 R}\right)}+R^{\eta}[\nabla g]_{C^{0, \eta}\left(\Delta_{2 R}\right)}
\end{array}\right\}
$$

where $C$ depends on $\mu, \tau, \kappa, d, p, \eta, M$ and $\rho$.

\section{Convergence rates}

Theorem 3.1. Let $\Omega$ be a $C^{1}$ domain. Suppose that $A$ satisfies $(1.2)-(1.3)$. Given $F \in L^{p}\left(\Omega ; \mathbb{R}^{d}\right)$ and $h \in W^{1, p}(\Omega)$ with $p>d$, and $g \in C^{0,1}\left(\partial \Omega ; \mathbb{R}^{d}\right)$ satisfying the compatibility condition (1.1), and we assume that $\left(u_{\varepsilon}, p_{\varepsilon}\right),\left(u_{0}, p_{0}\right)$ in $H^{1}\left(\Omega ; \mathbb{R}^{d}\right) \times L^{2}(\Omega)$ are weak solutions to Stokes systems $\left(D_{\varepsilon}\right)$, $\left(D_{0}\right)$, respectively. Then for any $(1 / 2)<\sigma<1$, we have

$\left\|u_{\varepsilon}-u_{0}\right\|_{L^{2}(\Omega)}+\left\|p_{\varepsilon}-p_{0}-\pi(\cdot / \varepsilon) S_{\varepsilon}\left(\psi_{2 \varepsilon} \nabla u_{0}\right)\right\|_{L^{2}(\Omega) / \mathbb{R}} \leq C \varepsilon^{\sigma-\frac{1}{2}}\left\{\|F\|_{L^{p}(\Omega)}+\|h\|_{W^{1, p}(\Omega)}+\|g\|_{C^{0,1}(\partial \Omega)}\right\}$

where $C$ depends only on $\mu, d, \sigma, p$ and $\Omega$.

Lemma 3.2. Assume the same conditions as in Theorem 3.1. Let $\left(u_{0}, p_{0}\right) \in H^{1}\left(\Omega ; \mathbb{R}^{d}\right) \times L^{2}(\Omega)$ be the weak solution to $\left(D_{0}\right)$. Then for any $\sigma \in(1 / 2,1)$, we have

$$
\left\|\nabla u_{0}\right\|_{L^{2}\left(\Omega \backslash \Sigma_{p_{1} \varepsilon}\right)} \leq C \varepsilon^{\sigma-\frac{1}{2}}\left\{\|F\|_{L^{p}(\Omega)}+\|h\|_{W^{1, p}(\Omega)}+\|g\|_{C^{0,1}(\partial \Omega)}\right\}
$$

and

$$
\left\|\nabla^{2} u_{0}\right\|_{L^{2}\left(\Sigma_{p_{2} \varepsilon}\right)} \leq C \varepsilon^{\sigma-\frac{3}{2}}\left\{\|F\|_{L^{p}(\Omega)}+\|h\|_{W^{1, p}(\Omega)}+\|g\|_{C^{0,1}(\partial \Omega)}\right\}
$$

where $p_{1}, p_{2}>0$ are fixed real number, and $C$ depends on $\mu, d, p_{1}, p_{2}, \sigma, p$ and $\Omega$.

Proof. We first handle the estimate (3.2). It is convenient to assume $\|F\|_{L^{p}(\Omega)}+\|h\|_{W^{1, p}(\Omega)}+$ $\|g\|_{C^{0,1}(\partial \Omega)}=1$. For any $x \in \Omega$, let $r=\delta(x)$. It follows from the interior Schauder estimates (2.12) that

$$
\begin{aligned}
&\left|\nabla u_{0}(x)\right| \leq C\left\{\frac{1}{r}\left(f_{B(x, r)}\left|u_{0}(y)-f_{B(x, r)} u_{0}\right|^{2} d y\right)^{\frac{1}{2}}+r\left(f_{B(x, r)}|F|^{p} d y\right)^{\frac{1}{p}}\right. \\
&\left.+r\left(f_{B(x, r)}|\nabla h|^{p} d y\right)^{\frac{1}{p}}+\|h\|_{L^{\infty}(B(x, r))}\right\} \\
& \leq C r^{\sigma-1}\left[u_{0}\right]_{C^{0, \sigma}(\Omega)}+C\left\{\|F\|_{L^{p}(\Omega)}+\|h\|_{W^{1, p}(\Omega)}\right\} \leq C r^{\sigma-1}
\end{aligned}
$$


where the global Hölder estimate (2.11) was used in the last inequality. We mention that the range of the Hölder exponent in $(2.11)$ is $(0,1)$. Then it follows from the co-area formula that

$$
\left\|\nabla u_{0}\right\|_{L^{2}\left(\Omega \backslash \Sigma_{p_{1} \varepsilon}\right)}^{2}=\int_{0}^{p_{1} \varepsilon} \int_{S_{t}}\left|\nabla u_{0}(y)\right|^{2} d S_{t}(y) d t \leq C \int_{0}^{p_{1} \varepsilon} \frac{d t}{t^{2 \sigma-2}} \leq C \varepsilon^{2 \sigma-1}
$$

whenever $\sigma \in(1 / 2,1)$. And hence this directly leads to (3.2).

Now we turn to show the estimate (3.3). Proceeding as in the proof of [22, Lemma 3.5], we let $u_{0}=v+w$ and $p_{0}=p_{0,1}+p_{0,2}$, and they satisfy

$$
\text { (i) }\left\{\begin{array} { r l r l } 
{ \mathcal { L } _ { 0 } ( v ) + \nabla p _ { 0 , 1 } } & { = \tilde { F } } & { \text { in } \mathbb { R } ^ { d } , } \\
{ \operatorname { d i v } ( v ) } & { = \tilde { h } } & { \text { in } \mathbb { R } ^ { d } , }
\end{array} \quad \text { and } \quad \text { (ii) } \left\{\begin{array}{rlrl}
\mathcal{L}_{0}(w)+\nabla p_{0,2} & =0 & & \text { in } \Omega, \\
\operatorname{div}(w) & =0 & & \text { in } \Omega, \\
w & =g-v & & \text { on } \partial \Omega,
\end{array}\right.\right.
$$

where $\tilde{F}$ is the extension of $F$ to $\mathbb{R}^{d}$ by 0 outside of $\Omega$, and $\tilde{h}$ is the $W^{1, q}$-extension of $h$ to $\mathbb{R}^{d}$ such that $\tilde{h}=h$ a.e. in $\Omega$ and $\|\tilde{h}\|_{W^{1, q}\left(\mathbb{R}^{d}\right)} \leq C\|h\|_{W^{1, q}(\Omega)}$. Due to the singular integral estimates (see [22, Lemma 3.4]) for Stokes system with constant coefficients, we have

$$
\left\|\nabla^{2} v\right\|_{L^{p}\left(\mathbb{R}^{d}\right)}+\|\nabla v\|_{L^{p}\left(\mathbb{R}^{d}\right)}+\left\|p_{0,1}\right\|_{W^{1, p}\left(\mathbb{R}^{d}\right)} \leq C\left\{\|F\|_{L^{p}(\Omega)}+\|h\|_{W^{1, p}(\Omega)}\right\} .
$$

To deal with the system (ii), as in the proof (3.4), we first obtain

$$
\begin{aligned}
|\nabla w(x)| & \leq \frac{C}{r}\left(f_{B(x, r / 2)}\left|w(y)-f_{B(x, r / 2)} w\right|^{2} d x\right)^{1 / 2} \leq C r^{\sigma-1}[w]_{C^{0, \sigma}(\Omega)} \\
& \leq C r^{\sigma-1}\left\{\|g\|_{C^{0,1}(\partial \Omega)}+\|v\|_{C^{0,1}(\partial \Omega)}\right\} \leq C r^{\sigma-1}\left\{\|g\|_{C^{0,1}(\partial \Omega)}+\|\nabla v\|_{W^{1, p}\left(\mathbb{R}^{d}\right)}\right\} \leq C r^{\sigma-1},
\end{aligned}
$$

where $p>d$, the fourth inequality above is a result of Sobolev imbedding theorem, and the last one follows from estimate (3.5). Also, it is clear to derive the following interior estimate

$$
\left|\nabla^{2} w(x)\right| \leq \frac{C}{r}\left(f_{B(x, r / 8)}|\nabla w|^{2} d y\right)^{\frac{1}{2}}
$$

Then we arrive at

$$
\int_{\Sigma_{p_{2} \varepsilon} \backslash \Sigma_{c_{0}}}\left|\nabla^{2} w\right|^{2} d x \leq C \int_{\Sigma_{p_{2} \varepsilon} \backslash \Sigma_{c_{0}}} f_{B(x, \delta(x) / 8)} \frac{|\nabla w(y)|^{2}}{[\delta(x)]^{2}} d y d x \leq C \int_{p_{2} \varepsilon}^{\infty} \frac{d t}{t^{4-2 \sigma}} \leq C \varepsilon^{2 \sigma-3}
$$

where we use the observation that $\delta(y) \approx \delta(x)$, and the estimate (3.6). This together with the estimates $\left\|\nabla^{2} v\right\|_{L^{2}\left(\Sigma_{p_{2} \varepsilon}\right)} \leq C$ and $\left\|\nabla^{2} w\right\|_{L^{2}\left(\Sigma_{c_{0}}\right)} \leq C$ gives

$$
\left\|\nabla^{2} u_{0}\right\|_{L^{2}\left(\Sigma_{p_{2} \varepsilon}\right)} \leq C \varepsilon^{\sigma-\frac{3}{2}}
$$

and the desired estimate (3.3) follows, and we have completed the proof.

Proof of Theorem 3.1. The following estimate can be derived by using the properties of smoothing operator and dual correctors as we stated in Section 2, details can be found in [22, Lemma 3.2], for example. 


$$
\begin{aligned}
&\left\|u_{\varepsilon}-u_{0}\right\|_{L^{2}(\Omega)}+\| p_{\varepsilon}-p_{0}-\pi(\cdot / \varepsilon) S_{\varepsilon}\left(\psi_{2 \varepsilon} \nabla u_{0}\right) \|_{L^{2}(\Omega) / \mathbb{R}} \\
& \leq C\left\{\left\|\nabla u_{0}\right\|_{L^{2}(\Omega \backslash 4 \varepsilon)}+\varepsilon\left\|\nabla^{2} u_{0}\right\|_{L^{2}\left(\Sigma_{2 \varepsilon}\right)}\right\},
\end{aligned}
$$

Substituting estimates (3.2) and (3.3) into the right hand side of the above estimate leads to the desired estimate (3.1), and the proof is complete.

Corollary 3.3. Assume the same conditions as in Theorem 3.1. If we additionally assume that the corrector $\pi$ is bounded, then we have

$$
\left\|p_{\varepsilon}-p_{0}-\pi(\cdot / \varepsilon) \psi_{2 \varepsilon} \nabla u_{0}\right\|_{L^{2}(\Omega) / \mathbb{R}} \leq C \varepsilon^{\sigma-\frac{1}{2}}\left\{\|F\|_{L^{p}(\Omega)}+\|h\|_{W^{1, p}(\Omega)}+\|g\|_{C^{0,1}(\partial \Omega)}\right\},
$$

where $\sigma \in(1 / 2,1)$ is given in Theorem 3.1, and $C$ depends on $\mu, d, \sigma, p, \eta, \tau, M$ and $\|\pi\|_{L^{\infty}(Y)}$.

Proof. The result follows from Theorem 3.1, Lemma 2.2 and Lemma 3.2.

Lemma 3.4. Let $\Omega$ be a bounded $C^{1, \tau}$ domain. Given $F \in L^{p}\left(D_{2} ; \mathbb{R}^{d}\right)$ and $h \in W^{1, p}\left(D_{2}\right)$ with $p>d$, and $g \in C^{1, \eta}\left(\Delta_{2}\right)$ with $g(0)=0$ and $\eta \in(0, \tau)$, let $\left(u_{0}, p_{0}\right) \in H^{1}\left(\Omega ; \mathbb{R}^{d}\right) \times L^{2}(\Omega)$ be the solution of $\mathcal{L}_{0}\left(u_{0}\right)+\nabla p_{0}=F$ and $\operatorname{div}\left(u_{0}\right)=h$ in $D_{2}$ with $u_{0}=u_{\varepsilon}$ on $\partial D_{2}$, where $\left(u_{\varepsilon}, p_{\varepsilon}\right)$ satisfies $\mathcal{L}_{\varepsilon}\left(u_{\varepsilon}\right)+\nabla p_{\varepsilon}=F$ and $\operatorname{div}\left(u_{\varepsilon}\right)=h$ in $D_{2}$, and $u_{\varepsilon}=g$ on $\Delta_{2}$. Then for any $5 \varepsilon \leq r<(1 / 4)$, there holds

$$
\begin{aligned}
\left(\frac{1}{\left|D_{r}\right|} \int_{D_{r} \backslash \Sigma_{4 \varepsilon}}\left|\nabla u_{0}\right|^{2} d x\right)^{\frac{1}{2}} & \leq C\left(\frac{\varepsilon}{r}\right)^{\frac{1}{2}}\left\{\left(f_{D_{1}}\left|u_{0}\right|^{2} d x\right)^{\frac{1}{2}}+\left(f_{D_{1}}|F|^{p} d x\right)^{\frac{1}{p}}\right. \\
& \left.+\left(f_{D_{1}}|\nabla h|^{p} d x\right)^{\frac{1}{p}}+\|h\|_{L^{\infty}\left(D_{1}\right)}+\|\nabla g\|_{C^{0, \eta}\left(\Delta_{1}\right)}\right\}
\end{aligned}
$$

and

$$
\begin{aligned}
\left(\frac{1}{\left|D_{r}\right|} \int_{D_{r} \cap \Sigma_{4 \varepsilon}}\left|\nabla^{2} u_{0}\right|^{2} d x\right)^{\frac{1}{2}} & \leq C\left(\frac{1}{\varepsilon r}\right)^{\frac{1}{2}}\left\{\left(f_{D_{2}}\left|u_{0}\right|^{2} d x\right)^{\frac{1}{2}}+\left(f_{D_{2}}|F|^{p} d x\right)^{\frac{1}{p}}\right. \\
& \left.+\left(f_{D_{2}}|\nabla h|^{p} d x\right)^{\frac{1}{p}}+\|h\|_{L^{\infty}\left(D_{2}\right)}+\|\nabla g\|_{C^{0, \eta}\left(\Delta_{2}\right)}\right\}
\end{aligned}
$$

where $C$ depends on $\mu, d, \eta, \tau, p$ and $M$.

Proof. The argument is quite similar to the one used in Lemma 3.2, and we will simply sketch the proof here. First, it is clear to see that the estimate (3.8) could be derived from

$$
\left\|\nabla u_{0}\right\|_{L^{\infty}\left(D_{1 / 2}\right)} \leq C\left\{\left(f_{D_{1}}\left|u_{0}\right|^{2} d x\right)^{\frac{1}{2}}+\left(f_{D_{1}}|F|^{p} d x\right)^{\frac{1}{p}}+\|h\|_{W^{1, p}\left(D_{1}\right)}+\|\nabla g\|_{C^{0, \eta}\left(\Delta_{1}\right)}\right\},
$$

which follows from the estimate (2.14). Once again, we let $u_{0}=v+w$ and $p_{0}=p_{0,1}+p_{0,2}$, which satisfy

$$
\text { (i) }\left\{\begin{array} { r l r l } 
{ \mathcal { L } _ { 0 } ( v ) + \nabla p _ { 0 , 1 } } & { = \tilde { F } } & { } & { \text { in } \mathbb { R } ^ { d } , } \\
{ \operatorname { d i v } ( v ) } & { = \tilde { h } } & { } & { \text { in } \mathbb { R } ^ { d } , }
\end{array} \quad \text { and } \quad \text { (ii) } \left\{\begin{array}{rlrl}
\mathcal{L}_{0}(w)+\nabla p_{0,2} & =0 & & \text { in } D_{2}, \\
\operatorname{div}(w) & =0 & & \text { in } D_{2}, \\
w & =u_{\varepsilon}-v & & \text { on } \partial D_{2} .
\end{array}\right.\right.
$$

Here $\tilde{F}$ and $\tilde{h}$ are the same extensions of $F$ and $h$ respectively as in the proof of Lemma 3.2. Also it follows from the local boundary estimate (2.14) that

$$
\begin{aligned}
\|\nabla w\|_{L^{\infty}\left(D_{1 / 2}\right)} & \leq C\left\{\|w\|_{L^{2}\left(D_{1}\right)}+\|\nabla g\|_{C^{0, \eta}\left(\Delta_{1}\right)}+\|\nabla v\|_{C^{0, \eta}\left(\Delta_{1}\right)}\right\} \\
& \leq C\left\{\left\|u_{0}\right\|_{L^{2}\left(D_{1}\right)}+\|\nabla g\|_{C^{0, \eta}\left(\Delta_{1}\right)}+\|F\|_{L^{p}\left(D_{2}\right)}+\|h\|_{W^{1, p}\left(D_{2}\right)}\right\}=: C_{D},
\end{aligned}
$$


where we use the Sobolev imbedding theorem and the estimate (3.5) in the last step, as well as the fact that $w=u_{0}-v$ in $D_{1}$ and $v-v(0)$ still satisfies (i). Then we have

$$
\int_{\Sigma_{4 \varepsilon} \cap D_{r}}\left|\nabla^{2} w\right|^{2} d x \leq C \int_{\Sigma_{4 \varepsilon} \cap D_{r}} f_{B(x, \delta(x) / 8)} \frac{|\nabla w(y)|^{2}}{[\delta(x)]^{2}} d y d x \leq C C_{D} r^{d-1} \int_{4 \varepsilon}^{r} \frac{d t}{t^{2}} \leq \frac{C C_{D} r^{d-1}}{\varepsilon}
$$

and this implies the estimate

$$
\begin{aligned}
\left(\frac{1}{\left|D_{r}\right|} \int_{D_{r} \cap \Sigma_{4 \varepsilon}}\left|\nabla^{2} w\right|^{2} d x\right)^{\frac{1}{2}} & \leq C\left(\frac{1}{\varepsilon r}\right)^{\frac{1}{2}}\left\{\left(f_{D_{1}}\left|u_{0}\right|^{2} d x\right)^{\frac{1}{2}}+\left(f_{D_{2}}|F|^{p} d x\right)^{\frac{1}{p}}\right. \\
& \left.+\left(f_{D_{2}}|\nabla h|^{p} d x\right)^{\frac{1}{p}}+\|h\|_{L^{\infty}\left(D_{2}\right)}+\|\nabla g\|_{C^{0, \eta}\left(\Delta_{2}\right)}\right\} .
\end{aligned}
$$

Moreover, using the estimate (3.5) again, we derive

$$
\left(\frac{1}{\left|D_{r}\right|} \int_{D_{r} \cap \Sigma_{4 \varepsilon}}\left|\nabla^{2} v\right|^{2} d x\right)^{\frac{1}{2}} \leq\left(f_{D_{r}}\left|\nabla^{2} v\right|^{p} d x\right)^{\frac{1}{p}} \leq C r^{-\frac{d}{p}}\left\{\left(f_{D_{2}}|F|^{p} d x\right)^{\frac{1}{p}}+\|h\|_{W^{1, p}\left(D_{2}\right)}\right\} .
$$

By noting that $p>d$ and $\varepsilon<r<1$, this together with (3.11) leads to the desired estimate (3.9), and we have completed the proof.

\section{$4 \quad$ Lipschitz estimates on velocity term}

We will use this section to provide the boundary Lipschitz estimate of the velocity, which is stated in the following theorem.

Theorem 4.1. Let $\Omega$ be a bounded $C^{1, \tau}$ domain. Suppose $A$ satisfies $(1.2)-(1.4)$. Let $\left(u_{\varepsilon}, p_{\varepsilon}\right) \in$ $H^{1}\left(D_{5} ; \mathbb{R}^{d}\right) \times L^{2}\left(D_{5}\right)$ be a weak solution of $\mathcal{L}_{\varepsilon}\left(u_{\varepsilon}\right)+\nabla p_{\varepsilon}=F$ and $\operatorname{div}\left(u_{\varepsilon}\right)=h$ in $D_{5}$ and $u_{\varepsilon}=g$ on $\Delta_{5}$, where $F \in L^{p}\left(D_{5} ; \mathbb{R}^{d}\right)$ and $h \in W^{1, p}\left(D_{5}\right)$ with $p>d$, and $g \in C^{1, \eta}\left(\Delta_{5} ; \mathbb{R}^{d}\right)$ with $g(0)=0$, and $0<\eta<\min \{\tau, 1-d / p\}$. Then there holds

$$
\begin{aligned}
\left(f_{D_{r}}\left|\nabla u_{\varepsilon}\right|^{2} d x\right)^{\frac{1}{2}} & \leq C\left\{\left(f_{D_{1}}\left|u_{\varepsilon}\right|^{2} d x\right)^{\frac{1}{2}}+\left(f_{D_{1}}|F|^{p} d x\right)^{\frac{1}{p}}\right. \\
& \left.+\left(f_{D_{1}}|\nabla h|^{p} d x\right)^{\frac{1}{p}}+\|h\|_{L^{\infty}\left(D_{1}\right)}+\|\nabla g\|_{C^{0, \eta}\left(\Delta_{1}\right)}\right\}
\end{aligned}
$$

for any $0<r<(1 / 4)$, where $C$ depends only on $\mu, \omega, d, M, \sigma$ and $p$.

Before we give the proof, we recall the following uniform boundary Hölder estimate of the velocity, obtained by compactness argument in [13, Theorem 6.1].

Theorem 4.2 (Boundary Hölder estimates). Let $\Omega$ be a bounded $C^{1}$ domain. Suppose A satisfies (1.2) and (1.3). Let $\left(u_{\varepsilon}, p_{\varepsilon}\right) \in H^{1}\left(D_{5} ; \mathbb{R}^{d}\right) \times L^{2}\left(D_{5}\right)$ be the weak solution to $\mathcal{L}_{\varepsilon}\left(u_{\varepsilon}\right)+\nabla p_{\varepsilon}=F$ and $\operatorname{div}\left(u_{\varepsilon}\right)=h$ in $D_{5}$, and $u_{\varepsilon}=g$ on $\Delta_{4}$, where $F \in L^{p}\left(D_{5} ; \mathbb{R}^{d}\right), h \in W^{1, p}\left(D_{5}\right)$ with $p>d$, and $g \in C^{0,1}\left(\Delta_{5} ; \mathbb{R}^{d}\right)$ with $g(0)=0$. Then for any $0<\sigma<1$, there holds

$$
\left(f_{D_{r}}\left|u_{\varepsilon}\right|^{2} d x\right)^{\frac{1}{2}} \leq C r^{\sigma}\left\{\left(f_{D_{1}}\left|u_{\varepsilon}\right|^{2} d x\right)^{\frac{1}{2}}+\|h\|_{W^{1, p}\left(D_{1}\right)}+\|\nabla g\|_{C^{0, \eta}\left(\Delta_{1}\right)}\right\}
$$

for any $\varepsilon \leq r<(1 / 4)$, where $C$ depends only on $\mu, p, d, \sigma, M$. 
Lemma 4.3. Let $\varepsilon \leq r<1$. Assume the same conditions as in Theorem 4.1. Let $\left(u_{\varepsilon}, p_{\varepsilon}\right) \in$ $H^{1}\left(D_{5} ; \mathbb{R}^{d}\right) \times L^{2}\left(D_{5}\right)$ be a weak solution of $\mathcal{L}_{\varepsilon}\left(u_{\varepsilon}\right)+\nabla p_{\varepsilon}=F$ and $\operatorname{div}\left(u_{\varepsilon}\right)=h$ in $D_{5}$, and $u_{\varepsilon}=g$ on $\Delta_{5}$. Then there exists $\left(u_{0}, p_{0}\right) \in H^{1}\left(D_{2} ; \mathbb{R}^{d}\right) \times L^{2}\left(D_{2}\right)$ such that $\mathcal{L}_{0}\left(u_{0}\right)+\nabla p_{0}=F$ and $\operatorname{div}\left(u_{0}\right)=h$ in $D_{2}$ and $u_{0}=g$ on $\Delta_{2}$, and for some $\lambda>0$, then

$$
\begin{aligned}
\left(f_{D_{r}}\left|u_{\varepsilon}-u_{0}\right|^{2} d x\right)^{\frac{1}{2}} \leq & C\left(\frac{\varepsilon}{r}\right)^{\lambda}\left\{\left(f_{D_{2 r}}\left|u_{\varepsilon}\right|^{2} d x\right)^{\frac{1}{2}}+r^{2}\left(f_{D_{2 r}}|F|^{p} d x\right)^{\frac{1}{p}}+r\|h\|_{L^{\infty}\left(D_{2 r}\right)}\right. \\
& \left.+r^{2}\left(f_{D_{2 r}}|\nabla h|^{p} d x\right)^{\frac{1}{p}}+r\|\nabla g\|_{L^{\infty}\left(\Delta_{2 r}\right)}+r^{1+\eta}[\nabla g]_{C^{0, \eta}\left(\Delta_{2 r}\right)}\right\}
\end{aligned}
$$

where $\lambda=2 \sigma-\frac{3}{2}$, and $C$ depends only on $\mu, \omega, \lambda, \tau, \eta, M, \sigma$ and $d$.

Proof. By rescaling argument, we may assume $r=1$. Since $\operatorname{div}\left(u_{\varepsilon}\right)=h$ in $D_{2}$, there exists $\left(u_{0}, p_{0}\right) \in$ $H^{1}\left(D_{2} ; \mathbb{R}^{d}\right) \times L^{2}\left(D_{2}\right)$ satisfying $\mathcal{L}_{0}\left(u_{0}\right)+\nabla p_{0}=F$, and $\operatorname{div}\left(u_{0}\right)=h$ in $D_{2}$, and $u_{0}=u_{\varepsilon}$ on $\partial D_{2}$. In view of Theorem 3.1, we have

$$
\left\|u_{\varepsilon}-u_{0}\right\|_{L^{2}\left(D_{1}\right)} \leq C \varepsilon^{\sigma-\frac{1}{2}}\left\{\|F\|_{L^{p}\left(D_{2}\right)}+\|h\|_{W^{1, p}\left(D_{2}\right)}+\|\nabla g\|_{L^{\infty}\left(\Delta_{2}\right)}+\left\|\nabla u_{\varepsilon}\right\|_{L^{\infty}\left(\partial D_{2} \backslash \Delta_{2}\right)}\right\},
$$

and it remains to estimate the last term in the right-hand side of (4.4). It is clear to see that $\partial D_{2} \backslash \Delta_{2}$ may be covered by $\left\{\tilde{D}_{4 \varepsilon}\right\}$ and $\left\{\tilde{B}_{\varepsilon}\right\}$. Hence it follows from the local estimate (2.14) that

$$
\begin{aligned}
\left\|\nabla u_{\varepsilon}\right\|_{L^{\infty}\left(\tilde{D}_{4 \varepsilon}\right)} \leq C\left\{\frac{1}{\varepsilon}\left(f_{\tilde{D}_{8 \varepsilon}}\left|u_{\varepsilon}\right|^{2} d x\right)^{\frac{1}{2}}+\varepsilon\left(f_{\tilde{D}_{8 \varepsilon}}|F|^{p} d x\right)^{\frac{1}{p}}+\varepsilon\left(f_{\tilde{D}_{8 \varepsilon}}|\nabla h|^{p} d x\right)^{\frac{1}{p}}\right. \\
\left.+\|h\|_{L^{\infty}\left(D_{4}\right)}+\|\nabla g\|_{L^{\infty}\left(\Delta_{4}\right)}+\varepsilon^{\eta}[\nabla g]_{C^{0, \eta}\left(\Delta_{4}\right)}\right\} \\
\leq C \varepsilon^{\sigma-1}\left\{\left(f_{D_{4}}\left|u_{\varepsilon}\right|^{2} d x\right)^{\frac{1}{2}}+\left(f_{D_{4}}|F|^{p} d x\right)^{\frac{1}{p}}+\|h\|_{W^{1, p}\left(D_{4}\right)}+\|\nabla g\|_{C^{0, \eta}\left(\Delta_{4}\right)}\right\},
\end{aligned}
$$

where the second inequality follows from the uniform Hölder estimate (4.2). Then for any $\tilde{B}_{\varepsilon}(x)$, where $x \in \partial D_{2} \backslash \Delta_{2}$, there are two cases: (1) $r=\operatorname{dist}\left(x, \Delta_{2}\right) \in[\varepsilon, 1 / 8]$ and $(2) r>\frac{1}{8}$. Obviously, the second case follows from the uniform interior Lipschitz estimates [13, Corollary 1.2] that

$$
\begin{aligned}
\left\|\nabla u_{\varepsilon}\right\|_{L^{\infty}\left(\tilde{B}_{\varepsilon}\right)} & \leq C\left\{\left(f_{\tilde{B}_{\frac{1}{4}}}\left|\nabla u_{\varepsilon}\right|^{2} d x\right)^{\frac{1}{2}}+\|h\|_{C^{0, \rho}\left(\tilde{B}_{\frac{1}{4}}\right)}+\|h\|_{L^{\infty}\left(\tilde{B}_{\frac{1}{4}}\right)}\right\} \\
& \leq C\left\{\left(f_{D_{4}}\left|u_{\varepsilon}\right|^{2} d x\right)^{\frac{1}{2}}+\left(f_{D_{4}}|F|^{p} d x\right)^{\frac{1}{p}}+\|h\|_{W^{1, p}\left(D_{4}\right)}+\|\nabla g\|_{L^{\infty}\left(\Delta_{4}\right)}\right\},
\end{aligned}
$$

where $\rho=1-d / p$, we use Caccippoli's inequality [13, Theorem 6.2] and the Sobolev imbedding theorem in the last inequality. We now turn to study the case (1):

$$
\begin{aligned}
\left\|\nabla u_{\varepsilon}\right\|_{L^{\infty}\left(\tilde{B}_{\varepsilon}\right)} & \leq\left\|\nabla u_{\varepsilon}\right\|_{L^{\infty}\left(\tilde{B}_{r / 2}\right)} \\
& \leq C\left\{\frac{1}{r}\left(f_{\tilde{D}_{2 r}}\left|u_{\varepsilon}\right|^{2} d x\right)^{\frac{1}{2}}+r\left(f_{\tilde{D}_{2 r}}|F|^{p} d x\right)^{\frac{1}{p}}+r\left(f_{\tilde{D}_{2 r}}|\nabla h|^{p} d x\right)^{\frac{1}{p}}\right. \\
& \left.+\|h\|_{L^{\infty}\left(D_{4}\right)}+\|\nabla g\|_{L^{\infty}\left(\Delta_{4}\right)}+r^{\eta}[\nabla g]_{C^{0, \eta}\left(\Delta_{4}\right)}\right\} \\
& \leq C \varepsilon^{\sigma-1}\left\{\left(f_{D_{4}}\left|u_{\varepsilon}\right|^{2} d x\right)^{\frac{1}{2}}+\left(f_{D_{4}}|F|^{p} d x\right)^{\frac{1}{p}}+\|h\|_{W^{1, p}\left(D_{4}\right)}+\|\nabla g\|_{C^{0, \eta}\left(\Delta_{4}\right)}\right\},
\end{aligned}
$$


where we use the uniform Hölder estimate (4.2), as well as the fact that $r>\varepsilon$, in the last inequality.

Consequently, combining (4.4), (4.5), (4.6) and (4.7), we have

$$
\left\|u_{\varepsilon}-u_{0}\right\|_{L^{2}\left(D_{1}\right)} \leq C \varepsilon^{\lambda}\left\{\left(f_{D_{4}}\left|\nabla u_{\varepsilon}\right|^{2} d x\right)^{\frac{1}{2}}+\left(f_{D_{4}}|F|^{p} d x\right)^{\frac{1}{p}}+\|h\|_{W^{1, p}\left(D_{4}\right)}+\|\nabla g\|_{C^{0, \eta}\left(\Delta_{4}\right)}\right\},
$$

where $\lambda=2 \sigma-\frac{3}{2}$. By rescaling argument we can derive the desired estimate (4.3), and we have completed the proof.

Before we proceed further, for any matrix $M \in \mathbb{R}^{d}$, we denote $G(r, v)$ as the following

$$
\begin{aligned}
G(r, v)=\frac{1}{r} & \inf _{M \in \mathbb{R}^{d \times d}}\left\{\left(f_{D_{r}}|v-M x|^{2} d x\right)^{\frac{1}{2}}+r^{2}\left(f_{D_{r}}|F|^{p} d x\right)^{\frac{1}{p}}+r\|h-\operatorname{Tr}(M)\|_{L^{\infty}\left(D_{r}\right)}\right. \\
& \left.+r^{2}\left(f_{D_{r}}|\nabla h|^{p} d x\right)^{\frac{1}{p}}+r\left\|\nabla_{T}(g-M x)\right\|_{L^{\infty}\left(\Delta_{r}\right)}+r^{1+\eta}\left[\nabla_{T}(g-M x)\right]_{C^{0, \eta}\left(\Delta_{r}\right)}\right\},
\end{aligned}
$$

where $\operatorname{Tr}(M)$ denotes the trace of $M$.

Lemma 4.4. Let $\left(u_{0}, p_{0}\right) \in H^{1}\left(D_{4} ; \mathbb{R}^{d}\right) \times L^{2}\left(D_{4}\right)$ be a solution of $\mathcal{L}_{0}\left(u_{0}\right)+\nabla p_{0}=F$ and $\operatorname{div}\left(u_{0}\right)=h$ in $D_{4}$, and $u_{0}=g$, where $g \in C^{0,1}\left(\Delta_{4}\right)$ with $g(0)=0$. Then there exists $\theta \in(0,1 / 4)$, depending on $\mu, d, \eta$ and $M$, such that

$$
G\left(\theta r, u_{0}\right) \leq \frac{1}{2} G\left(r, u_{0}\right)
$$

holds for any $r \in(0,1)$.

Proof. We may assume $r=1$ by rescaling argument. By the definition of $G\left(\theta, u_{0}\right)$, we see that

$$
\begin{aligned}
G\left(\theta, u_{0}\right) \leq & \frac{1}{\theta}\left\{\left(f_{D_{\theta}}\left|u_{0}-M_{0} x\right|^{2} d x\right)^{\frac{1}{2}}+\theta^{2}\left(f_{D_{\theta}}|F|^{p} d x\right)^{\frac{1}{p}}+\theta\left\|h-\operatorname{Tr}\left(M_{0}\right)\right\|_{L^{\infty}\left(D_{\theta}\right)}\right. \\
& \left.+\theta^{2}\left(f_{D_{\theta}}|\nabla h|^{p} d x\right)^{\frac{1}{p}}+\theta\left\|\nabla_{T}\left(g-M_{0} x\right)\right\|_{L^{\infty}\left(\Delta_{\theta}\right)}+\theta^{1+\eta}\left[\nabla_{T}\left(g-M_{0} x\right)\right]_{C^{0, \eta}\left(\Delta_{\theta}\right)}\right\} \\
\leq & \theta^{\eta}\left\{\left[\nabla u_{0}\right]_{C^{0, \eta}\left(D_{1 / 2}\right)}+\left(f_{D_{1 / 2}}|F|^{p} d x\right)^{\frac{1}{p}}+\left(f_{D_{1 / 2}}|\nabla h|^{p} d x\right)^{\frac{1}{p}}\right\},
\end{aligned}
$$

where we choose $M_{0}=\nabla u_{0}(0)$. For any $M \in \mathbb{R}^{d \times d}$, we let $\tilde{u}_{0}=u_{0}-M x$. Clearly it satisfies the system: $\mathcal{L}_{0}\left(\tilde{u}_{0}\right)+\nabla p_{0}=F$, and $\operatorname{div}\left(\tilde{u}_{0}\right)=h-\operatorname{Tr}(M)$ in $D_{4}, \tilde{u}_{0}=g-M x$ on $\Delta_{4}$. Hence it follows from boundary Schauder estimates (2.13) that

$$
\left[\nabla u_{0}\right]_{C^{0, \eta}\left(D_{1 / 2}\right)}=\left[\nabla \tilde{u}_{0}\right]_{C^{0, \eta}\left(D_{1 / 2}\right)} \leq C G\left(1, u_{0}\right) .
$$

It is clear to see that there exists $\theta \in(0,1 / 4)$ such that $G\left(\theta, u_{0}\right) \leq \frac{1}{2} G\left(1, u_{0}\right)$. Then the desire result (4.9) can be obtained simply by a rescaling argument.

For simplicity, we also denote $\Phi(r)$ by

$$
\begin{aligned}
\Phi(r)=\frac{1}{r}\left\{\left(f_{D_{r}}\left|u_{\varepsilon}\right|^{2} d x\right)^{\frac{1}{2}}\right. & +r^{2}\left(f_{D_{r}}|F|^{p} d x\right)^{\frac{1}{p}}+r\|h\|_{L^{\infty}\left(D_{r}\right)} \\
& \left.+r^{2}\left(f_{D_{r}}|\nabla h|^{p} d x\right)^{\frac{1}{p}}+r\|\nabla g\|_{L^{\infty}\left(\Delta_{2 r}\right)}+r^{1+\eta}[\nabla g]_{C^{0, \eta}\left(\Delta_{r}\right)}\right\} .
\end{aligned}
$$


Lemma 4.5. Let $\lambda$ be given in Lemma 4.3. Assume the same conditions as in Theorem 4.1. Let $\left(u_{\varepsilon}, p_{\varepsilon}\right)$ be the solution of $\mathcal{L}_{\varepsilon}\left(u_{\varepsilon}\right)+\nabla p_{\varepsilon}=F$ and $\operatorname{div}\left(u_{\varepsilon}\right)=h$ in $D_{5}$ with $u_{\varepsilon}=g$ on $\Delta_{5}$. Then we have

$$
G\left(\theta r, u_{\varepsilon}\right) \leq \frac{1}{2} G\left(r, u_{\varepsilon}\right)+C\left(\frac{\varepsilon}{r}\right)^{\lambda} \Phi(2 r)
$$

for any $r \in[\varepsilon, 1 / 2]$, where $\theta \in(0,1 / 4)$ is given in Lemma 4.4 .

Proof. Fix $r \in[\varepsilon, 1 / 2]$, let $\left(u_{0}, p_{0}\right)$ be a solution to $\mathcal{L}_{0}\left(u_{0}\right)+\nabla p_{0}=F$ and $\operatorname{div}\left(u_{0}\right)=h$ in $D_{r}$, and $u_{0}=u_{\varepsilon}$ on $\partial D_{r}$. Then we have

$$
\begin{aligned}
G\left(\theta r, u_{\varepsilon}\right) & \leq \frac{1}{\theta r}\left(f_{D_{\theta r}}\left|u_{\varepsilon}-u_{0}\right|^{2} d x\right)^{\frac{1}{2}}+G\left(\theta r, u_{0}\right) \\
& \leq \frac{C}{r}\left(f_{D_{r}}\left|u_{\varepsilon}-u_{0}\right|^{2} d x\right)^{\frac{1}{2}}+\frac{1}{2} G\left(r, u_{0}\right) \\
& \leq \frac{1}{2} G\left(r, u_{\varepsilon}\right)+\frac{C}{r}\left(f_{D_{r}}\left|u_{\varepsilon}-u_{0}\right|^{2} d x\right)^{\frac{1}{2}} \\
& \leq \frac{1}{2} G\left(r, u_{\varepsilon}\right)+C(\varepsilon / r)^{\lambda} \Phi(2 r),
\end{aligned}
$$

where we use the estimate (4.9) in the second inequality, and (4.3) in the last one. The proof is complete.

Lemma 4.6. Let $\Psi(r)$ and $\psi(r)$ be two nonnegative continuous functions on the integral $(0,1]$. Let $0<\varepsilon<\frac{1}{4}$. Suppose that there exists a constant $C_{0}$ such that

$$
\left\{\begin{array}{l}
\max _{r \leq t \leq 2 r} \Psi(r) \leq C_{0} \Psi(2 r) \\
\max _{r \leq s, t \leq 2 r}|\psi(r)-\psi(s)| \leq C_{0} \Psi(2 r)
\end{array}\right.
$$

for any $r \in[\varepsilon, 1 / 2]$. We further assume that

$$
\Psi(\theta r) \leq \frac{1}{2} \Psi(r)+C_{0} w(\varepsilon / r)\{\Psi(2 r)+\psi(2 r)\}
$$

holds for any $r \in[\varepsilon, 1 / 2]$, where $\theta \in(0,1 / 4)$ and $\omega$ is a nonnegative increasing function in $[0,1]$ such that $\omega(0)=0$ and

$$
\int_{0}^{1} \frac{w(t)}{t} d t<\infty
$$

Then, we have

$$
\max _{\varepsilon \leq r \leq 1}\{\Psi(r)+\psi(r)\} \leq C\{\Psi(1)+\psi(1)\},
$$

where $C$ depends only on $C_{0}, \theta$ and $w$.

Proof. See [17, Lemma 8.5].

Proof of Theorem 4.1. It is fine to assume $0<\varepsilon<1 / 4$, otherwise it follows from the classical theory. In view of Lemma 4.6, we set $\Psi(r)=G\left(r, u_{\varepsilon}\right), w(t)=t^{\lambda}$, where $\lambda>0$ is given in Lemma 
4.3. In order to prove the desired estimate (4.1), it is sufficient to verify (4.11) and (4.12). Let $\psi(r)=\left|M_{r}\right|$, where $M_{r}$ is the matrix associated with $\Psi(r)$, i.e., in the following sense,

$$
\begin{aligned}
\Psi(r)=\frac{1}{r}\{ & \left(f_{D_{r}}\left|u_{\varepsilon}-M_{r} x\right|^{2} d x\right)^{\frac{1}{2}}+r^{2}\left(f_{D_{r}}|F|^{p} d x\right)^{\frac{1}{p}}+r\left\|h-\operatorname{Tr}\left(M_{r}\right)\right\|_{L^{\infty}\left(D_{r}\right)} \\
& \left.+r^{2}\left(f_{D_{r}}|\nabla h|^{p} d x\right)^{\frac{1}{p}}+r\left\|\nabla_{T}\left(g-M_{r} x\right)\right\|_{L^{\infty}\left(\Delta_{r}\right)}+r^{1+\eta}\left[\nabla_{T}\left(g-M_{r} x\right)\right]_{C^{0, \eta}\left(\Delta_{r}\right)}\right\},
\end{aligned}
$$

Then we have,

$$
\Phi(2 r) \leq \Psi(2 r)+\psi(2 r) .
$$

This together with Lemma 4.5 gives

$$
\Psi(\theta r) \leq \frac{1}{2} \Psi(r)+C_{0} w(\varepsilon / r)\{\Psi(2 r)+\psi(2 r)\},
$$

which satisfies the condition (4.12) in Lemma 4.6. Let $t, s \in[r, 2 r]$, and $v(x)=\left(M_{t}-M_{s}\right) x$. It is clear to see $v$ is harmonic in $\mathbb{R}^{d}$. Since $D_{r}$ satisfies the interior ball condition, we arrive at

$$
\begin{aligned}
\left|M_{t}-M_{s}\right| & \leq \frac{C}{r}\left(f_{D_{r}}\left|\left(M_{t}-M_{s}\right) x\right|^{2} d x\right)^{\frac{1}{2}} \\
& \leq \frac{C}{t}\left(f_{D_{t}}\left|u_{\varepsilon}-M_{t} x\right|^{2} d x\right)^{\frac{1}{2}}+\frac{C}{s}\left(f_{D_{s}}\left|u_{\varepsilon}-M_{s} x\right|^{2} d x\right)^{\frac{1}{2}} \\
& \leq C\{\Psi(t)+\Psi(s)\} \leq C \Psi(2 r),
\end{aligned}
$$

where the second and the last steps are based on the fact that $s, t \in[r, 2 r]$. Due to the same reason, it is easy to obtain $\Psi(r) \leq C \Psi(2 r)$, and the estimate (4.15) admits the condition (4.11). Besides, $w$ here obviously satisfies the condition (4.13). Hence, according to Lemma 4.6, for any $r \in[\varepsilon, 1 / 4]$, we have the following estimate

$$
\frac{1}{r}\left(f_{D_{2 r}}\left|u_{\varepsilon}\right|^{2} d x\right)^{\frac{1}{2}} \leq C\{\Psi(2 r)+\psi(2 r)\} \leq C\{\Psi(1)+\psi(1)\} .
$$

Hence, for $\varepsilon \leq r<(1 / 4)$, the desired estimate (4.1) consequently follows from (4.16) and the Cacciopoli's inequality [13, Theorem 6.2]. Obviously, the case of $0<r<\varepsilon$ can be done simply by the blow-up argument, and we have completed the proof.

\section{$5 \quad L^{\infty}$ estimates on pressure term}

Now we move on to provide the boundary $L^{\infty}$ estimates on the pressure term.

Theorem 5.1. Assume the same conditions as in Theorem 1.2. Let $\left(u_{\varepsilon}, p_{\varepsilon}\right) \in H^{1}\left(D_{5} ; \mathbb{R}^{d}\right) \times L^{2}\left(D_{5}\right)$ be the solution of $\mathcal{L}_{\varepsilon}\left(u_{\varepsilon}\right)+\nabla p_{\varepsilon}=F$ and $\operatorname{div}\left(u_{\varepsilon}\right)=h$ in $D_{5}$ with $u_{\varepsilon}=g$ on $\Delta_{5}$, where $F \in L^{p}\left(D_{5} ; \mathbb{R}^{d}\right)$ and $h \in W^{1, p}\left(D_{5}\right)$ with $p>d$, and $g \in C^{1, \eta}$ with $g(0)=0$ and $\eta \in(0, \tau]$. Then there holds

$$
\begin{aligned}
\left|f_{D_{r}} p_{\varepsilon}-f_{D_{1}} p_{\varepsilon} d x\right| & \leq C\left\{\left(f_{D_{2}}\left|u_{\varepsilon}\right|^{2} d x\right)^{\frac{1}{2}}\right. \\
& \left.+\left(f_{D_{2}}|F|^{p} d x\right)^{\frac{1}{p}}+\left(f_{D_{2}}|\nabla h|^{p} d x\right)^{\frac{1}{p}}+\|h\|_{L^{\infty}\left(D_{2}\right)}+\|\nabla g\|_{C^{0, \eta}\left(\Delta_{2}\right)}\right\}
\end{aligned}
$$

for any $0<r<(1 / 4)$, where the constant $C$ depends on $\mu, d, \eta, M$ and $p$. 
Lemma 5.2. Let $\left(u_{\varepsilon}, p_{\varepsilon}\right)$ be the solution of $\mathcal{L}_{\varepsilon}\left(u_{\varepsilon}\right)+\nabla p_{\varepsilon}=F, \operatorname{div}\left(u_{\varepsilon}\right)=h$ in $D_{5}$, and $u_{\varepsilon}=g$ on $\Delta_{5}$. Then there exists $\left(u_{0}, p_{0}\right) \in H^{1}\left(D_{2} ; \mathbb{R}^{d}\right) \times L^{2}\left(D_{2}\right)$ such that $\mathcal{L}_{0}\left(u_{0}\right)+\nabla p_{0}=F$ and $\operatorname{div}\left(u_{0}\right)=h$ in $D_{2}$ with $u_{0}=g$ on $\Delta_{2}$, and there holds

$$
\begin{gathered}
\left(f_{D_{r}}\left|p_{\varepsilon}-p_{0}-\pi(\cdot / \varepsilon) \psi_{4 \varepsilon} \nabla u_{0}-c\right|^{2} d x\right)^{\frac{1}{2}} \leq C\left(\frac{\varepsilon}{r}\right)^{\lambda}\left\{\frac{1}{r}\left(f_{D_{4 r}}\left|u_{\varepsilon}\right|^{2} d x\right)^{\frac{1}{2}}\right. \\
\left.+\|h\|_{L^{\infty}\left(D_{4 r}\right)}+r\left(f_{D_{4 r}}|\nabla h|^{p} d x\right)^{\frac{1}{p}}+r\left(f_{D_{4 r}}|F|^{p} d x\right)^{\frac{1}{p}}+\|\nabla g\|_{L^{\infty}\left(\Delta_{4 r}\right)}+r^{\eta}[\nabla g]_{C^{0, \eta}\left(\Delta_{4 r}\right)}\right\},
\end{gathered}
$$

for any $\varepsilon \leq r<(1 / 4)$, where $\lambda=2 \sigma-3 / 2$, and

$$
c=f_{D_{r}}\left[p_{\varepsilon}-p_{0}-\pi(\cdot / \varepsilon) \psi_{4 \varepsilon} \nabla u_{0}\right] d x
$$

and $C$ depends only on $\mu, d, \eta$ and $M$.

Proof. This lemma as the counterpart of Lemma 4.3 obeys a similar proof. By rescaling argument, we may prove it for $r=1$. Let $\left(u_{0}, p_{0}\right)$ be the same one given in Lemma 4.3. Hence, it follows from the estimate (3.7) that

$$
\begin{aligned}
\left\|p_{\varepsilon}-p_{0}-\pi(\cdot / \varepsilon) \psi_{2 \varepsilon} \nabla u_{0}\right\|_{L^{2}\left(D_{1}\right) / \mathbb{R}} & \leq\left\|p_{\varepsilon}-p_{0}-\pi(\cdot / \varepsilon) \psi_{2 \varepsilon} \nabla u_{0}\right\|_{L^{2}\left(D_{2}\right) / \mathbb{R}} \\
& \leq C \varepsilon^{\sigma-\frac{1}{2}}\left\{\|F\|_{L^{p}\left(D_{2}\right)}+\|h\|_{W^{1, p}\left(D_{2}\right)}+\|\nabla g\|_{L^{\infty}\left(\Delta_{2}\right)}+\left\|\nabla u_{\varepsilon}\right\|_{L^{\infty}\left(\partial D_{2} \backslash \Delta_{2}\right)}\right\} .
\end{aligned}
$$

This together with the estimates (4.5), (4.6) and (4.7) gives

$$
\left\|p_{\varepsilon}-p_{0}-\pi(\cdot / \varepsilon) \psi_{2 \varepsilon} \nabla u_{0}\right\|_{L^{2}\left(D_{1}\right) / \mathbb{R}} \leq C \varepsilon^{\lambda}\left\{\left\|u_{\varepsilon}\right\|_{L^{2}\left(D_{4}\right)}+\|F\|_{L^{p}\left(D_{4}\right)}+\|h\|_{W^{1, p}\left(D_{4}\right)}+\|\nabla g\|_{C^{0, \eta}\left(\Delta_{4}\right)}\right\},
$$

where $\lambda=2 \sigma-3 / 2$. The desired estimate (5.2) is derived from the rescaling argument for any $r \in[\varepsilon, 1 / 4]$.

Lemma 5.3. Assume the same conditions as in Theorem 1.2. Let $\left(u_{0}, p_{0}\right) \in H^{1}\left(D_{4} ; \mathbb{R}^{d}\right) \times L^{2}\left(D_{4}\right)$ be the weak solution of $\mathcal{L}_{0}\left(u_{0}\right)+\nabla p_{0}=F$ and $\operatorname{div}\left(u_{0}\right)=h$ in $D_{4}$ with $u_{0}=g$ on $\Delta_{4}$. Then for any $0<\rho<\min \{\eta, 1-d / p\}$, there holds

$$
\left(f_{D_{r}}\left|p_{0}-f_{D_{s}} p_{0}\right|^{2} d x\right)^{\frac{1}{2}} \leq C s^{\rho}\left\{\left\|u_{0}\right\|_{L^{2}\left(D_{2}\right)}+\|F\|_{L^{p}\left(D_{2}\right)}+\|h\|_{W^{1, p}\left(D_{2}\right)}+\|\nabla g\|_{C^{0, \eta}\left(\Delta_{2}\right)}\right\}
$$

for any $0<r \leq s \leq 1$, where $C$ depends only on $\mu, d, p, \eta, M$ and $\rho$.

Proof. Since $\mathcal{L}_{0}$ is an operator with constant coefficients, it is well known that $\left(u_{0}, p_{0}\right) \in C^{1, \rho}\left(D_{1} ; \mathbb{R}^{d}\right) \times$ $C^{0, \rho}\left(D_{1}\right)$, and it follows from the local estimate (2.13) that

$$
\begin{aligned}
f_{D_{r}}\left|p_{0}-f_{D_{s}} p_{0}\right|^{2} d x & =f_{D_{r}}\left|p_{0}-p_{0}(0)-f_{D_{s}}\left[p_{0}-p_{0}(0)\right] d y\right|^{2} d x \\
& \leq C\left\{r^{2 \rho}+s^{2 \rho}\right\}\left[p_{0}\right]_{C^{0, \rho}\left(D_{1}\right)}^{2} \\
& \leq C s^{2 \rho}\left\{\left\|u_{0}\right\|_{L^{2}\left(D_{2}\right)}+\|F\|_{L^{p}\left(D_{2}\right)}+\|h\|_{W^{1, p}\left(D_{2}\right)}+\|\nabla g\|_{C^{0, \eta}\left(\Delta_{2}\right)}\right\} .
\end{aligned}
$$

This implies the desired estimate (5.4). We have completed the proof. 
Proof of Theorem 5.1. For any $\varepsilon \leq r<(1 / 4)$, there exists an integer $k>0$ such that $2^{-k-1} \leq r<2^{-k}$. Then we have

$$
\left|f_{D_{r}} p_{\varepsilon}-f_{D_{1}} p_{\varepsilon} d x\right| \leq 2\left|f_{D_{2^{-k}}} p_{\varepsilon}-f_{D_{1}} p_{\varepsilon} d x\right| \leq 4 \sum_{j=1}^{k}\left|f_{D_{2^{-j}}} p_{\varepsilon}-f_{D_{2^{-j+1}}} p_{\varepsilon} d x\right|
$$

It now remains to estimate each terms above. For simplicity, we denote $Z_{\varepsilon}$ and its average by

$$
Z_{\varepsilon}(x)=p_{\varepsilon}(x)-p_{0}(x)-\pi_{i}^{\gamma}(x / \varepsilon) \psi_{4 \varepsilon}(x) \nabla_{i} u_{0}^{\gamma}(x), \quad \overline{(Z}_{D_{D^{-j}}}=f_{D_{2^{-j}}} Z_{\varepsilon}(x) d x .
$$

Thus for any $c \in \mathbb{R}$, it follows that

$$
\begin{gathered}
\left|f_{D_{2^{-j}}} p_{\varepsilon}-f_{D_{2^{-j+1}}} p_{\varepsilon} d x\right| \leq \mid \\
f_{D_{2^{-j}}}\left\{\left[Z_{\varepsilon}-c-f_{D_{2^{-j+1}}}\left(Z_{\varepsilon}-c\right) d y\right]+\left[p_{0}(x)-f_{D_{2^{-j}+1}} p_{0} d y\right]\right. \\
\left.+\left[\pi(\cdot / \varepsilon) \psi_{4 \varepsilon} \nabla u_{0}-f_{D_{2^{-j+1}}} \pi(y / \varepsilon) \psi_{4 \varepsilon} \nabla u_{0} d y\right]\right\} d x \mid .
\end{gathered}
$$

Now if we set $c=\overline{(Z \varepsilon}_{D_{2^{-j+1}}}$, then the right-hand side of (5.6) is controlled by

$$
\begin{aligned}
\left(f_{D_{2^{-j}}} \mid Z_{\varepsilon}\right. & \left.-{\overline{\left(Z_{\varepsilon}\right)_{D_{2^{-j+1}}}}}^{2} d x\right)^{\frac{1}{2}}+\left(f_{D_{2^{-j}}}\left|p_{0}-f_{D_{2^{-j+1}}} p_{0}\right|^{2} d x\right)^{\frac{1}{2}} \\
& +\left|f_{D_{2^{-j}}}\left[\pi(\cdot / \varepsilon) \psi_{4 \varepsilon} \nabla u_{0}-f_{D_{2^{-j+1}}} \pi(y / \varepsilon) \psi_{4 \varepsilon} \nabla u_{0} d y\right] d x\right|=: I_{1}+I_{2}+I_{3} .
\end{aligned}
$$

We first handle $I_{1}$, and it follows that

$$
\begin{aligned}
& I_{1} \leq C\left(f_{D_{2^{-j+1}}}\left|Z_{\varepsilon}-{\overline{\left(Z_{\varepsilon}\right)}}_{D_{2^{-j+1}}}\right|^{2} d x\right)^{\frac{1}{2}} \\
& \leq C\left(\frac{\varepsilon}{2^{-j}}\right)^{\lambda}\left\{\frac{1}{2^{-j}}\left(f_{D_{2^{-j}}}\left|u_{\varepsilon}\right|^{2} d x\right)^{\frac{1}{2}}+2^{-j}\left(f_{D_{2^{-j}}}|F|^{p} d x\right)^{\frac{1}{p}}\right. \\
& \left.+2^{-j}\left(f_{D_{2^{-j}}}|\nabla h|^{p} d x\right)^{\frac{1}{p}}+\|h\|_{L^{\infty}\left(D_{2}\right)}+\|\nabla g\|_{C^{0, \eta}\left(\Delta_{2}\right)}\right\} \\
& \leq C\left(\varepsilon 2^{j}\right)^{\lambda}\left\{\left(f_{D_{2}}\left|u_{\varepsilon}\right|^{2} d x\right)^{\frac{1}{2}}+\|F\|_{L^{p}\left(D_{2}\right)}+\|\nabla h\|_{L^{p}\left(D_{2}\right)}+\|h\|_{L^{\infty}\left(D_{2}\right)}+\|\nabla g\|_{C^{0, \eta}\left(\Delta_{2}\right)}\right\},
\end{aligned}
$$

where we use the Lipschitz estimate (4.16) in the last step, as well as the fact $p>d$. Then we proceed to study $I_{2}$, and it follows from the estimate (5.4) that

$$
I_{2} \leq C\left(2^{-j}\right)^{\rho}\left\{\left\|u_{0}\right\|_{L^{2}\left(D_{2}\right)}+\|F\|_{L^{p}\left(D_{2}\right)}+\|h\|_{W^{1, p}\left(D_{2}\right)}+\|\nabla g\|_{C^{0, \eta}\left(\Delta_{2}\right)}\right\} .
$$

We now investigate $I_{3}$. In the case of $\varepsilon \leq 2^{-j+1}<3 \varepsilon$, there is nothing to do since the term $S_{\varepsilon}\left(\psi_{4 \varepsilon} \nabla u_{0}\right)$ is supported outside $D_{2^{-j+1}}$. Hence we only deal with $I_{3}$ in the case of $2^{-j} \geq 3 \varepsilon$.

$$
\begin{aligned}
I_{3} & \leq\left|f_{D_{2^{-j}}}[\pi(\cdot / \varepsilon)-\widehat{\pi}] \psi_{4 \varepsilon} \nabla u_{0} d x\right|+\left|f_{D_{2^{-j+1}}}[\pi(\cdot / \varepsilon)-\widehat{\pi}] \psi_{4 \varepsilon} \nabla u_{0} d x\right| \\
& +|\widehat{\pi}|\left|f_{D_{2^{-j}}}\left(\psi_{4 \varepsilon} \nabla u_{0}-f_{D_{2^{-j}+1}} \psi_{4 \varepsilon} \nabla u_{0} d y\right) d x\right|=: I_{31}+I_{32}+I_{33},
\end{aligned}
$$


where $\widehat{\pi}=f_{Y} \pi(y) d y$. Note that $I_{31}$ and $I_{32}$ obey the same computations. Taking $I_{31}$ for example, there exists $V \in H_{p e r}^{1}\left(Y ; \mathbb{R}^{d}\right)$ such that $\operatorname{div}_{y}(V)=\pi(y)-\widehat{\pi}$ in $\mathbb{R}^{d}$. Thus we have

$$
\begin{aligned}
I_{31} & =\left|f_{D_{2^{-j}}}[\pi(\cdot / \varepsilon)-\widehat{\pi}] \psi_{4 \varepsilon} \nabla u_{0} d x\right|=\varepsilon\left|f_{D_{2^{-j}}} \operatorname{div}_{x}[V(x / \varepsilon)] \psi_{4 \varepsilon} \nabla u_{0} d x\right| \\
& \leq \varepsilon f_{D_{2^{-j}}}\left|V(x / \varepsilon) \cdot \nabla\left(\psi_{4 \varepsilon} \nabla u_{0}\right)\right| d x+\frac{C \varepsilon}{2^{-j}}\left(f_{\partial\left(D_{2^{-j}}\right)}\left|V(x / \varepsilon) \psi_{4 \varepsilon} \nabla u_{0}\right|^{2} d x\right)^{\frac{1}{2}} \\
& \leq \frac{C}{\left|D_{2^{-j}}\right|^{\frac{1}{2}}}\left\{\left\|\nabla u_{0}\right\|_{L^{2}\left(D_{\left.2^{-j} \backslash \Sigma_{4 \varepsilon}\right)}+\varepsilon\left\|\nabla^{2} u_{0}\right\|_{L^{2}\left(D_{2^{-j}} \cap \Sigma_{4 \varepsilon}\right)}\right\}+C \varepsilon 2^{j}\left\|\nabla u_{0}\right\|_{L^{\infty}\left(D_{1 / 2}\right)}}\right. \\
& \leq C\left\{\left(\varepsilon 2^{j}\right)^{\frac{1}{2}}+\left(\varepsilon 2^{j}\right)\right\}\left\{\left(f_{D_{2}}\left|u_{0}\right|^{2} d x\right)^{\frac{1}{2}}+\|F\|_{L^{p}\left(D_{2}\right)}+\|h\|_{W^{1, p}\left(D_{2}\right)}+\|\nabla g\|_{C^{0, \eta}\left(\Delta_{2}\right)}\right\},
\end{aligned}
$$

where we use Remark 2.5 in the second inequality, and the estimates (3.8), (3.9) and (2.14) in the last one. We now turn to estimate $I_{33}$. It follows that

$$
\begin{aligned}
I_{33} & =|\widehat{\pi}|\left|f_{D_{2^{-j}}}\left(\psi_{4 \varepsilon} \nabla u_{0}-f_{D_{2^{-j+1}}} \psi_{4 \varepsilon} \nabla u_{0} d y\right) d x\right| \leq C f_{D_{2^{-j}}}\left|\psi_{4 \varepsilon} \nabla u_{0}-\nabla u_{0}(0)\right| d x \\
& \leq C f_{D_{2^{-j}}}\left|\psi_{4 \varepsilon}\left(\nabla u_{0}-\nabla u_{0}(0)\right)+\left(\psi_{4 \varepsilon}-1\right) \nabla u_{0}(0)\right| d x \\
& \leq C\left(2^{-j}\right)^{\rho}\left[\nabla u_{0}\right]_{C^{0, \rho}\left(D_{1}\right)}+C\left(\varepsilon 2^{j}\right)\left\|\nabla u_{0}\right\|_{L^{\infty}\left(D_{1}\right)} \\
& \leq C\left\{\left(2^{-j}\right)^{\rho}+\left(\varepsilon 2^{j}\right)\right\}\left\{\left\|u_{0}\right\|_{L^{2}\left(D_{2}\right)}+\|F\|_{L^{p}\left(D_{2}\right)}+\|h\|_{W^{1, p}\left(D_{2}\right)}+\|\nabla g\|_{C^{0, \eta}\left(\Delta_{2}\right)}\right\},
\end{aligned}
$$

where we employ the estimate (2.14) and (2.13) in the last step. Moreover, it is clear to see that the estimates (5.9) and (5.10) give

$$
I_{3} \leq C\left\{\left(\varepsilon 2^{j}\right)+\left(\varepsilon 2^{j}\right)^{\frac{1}{2}}+\left(2^{-j}\right)^{\rho}\right\}\left\{\left(f_{D_{2}}\left|u_{0}\right|^{2} d x\right)^{\frac{1}{2}}+\|F\|_{L^{p}\left(D_{2}\right)}+\|h\|_{W^{1, p}\left(D_{2}\right)}+\|\nabla g\|_{C^{0, \eta}\left(\Delta_{2}\right)}\right\} .
$$

Combining the estimates (5.7), (5.8) and (5.11), we obtain that

$$
\begin{aligned}
& \left|f_{D_{r}} p_{\varepsilon}-f_{D_{1}} p_{\varepsilon} d x\right| \leq \sum_{j=1}^{k}\left(\varepsilon 2^{j}\right)^{\frac{1}{2}}\left\{\left(f_{D_{2}}\left|u_{\varepsilon}\right|^{2} d x\right)^{\frac{1}{2}}+\|F\|_{L^{p}\left(D_{2}\right)}+\|h\|_{W^{1, p}\left(D_{2}\right)}+\|\nabla g\|_{C^{0, \eta}\left(\Delta_{2}\right)}\right\} \\
& \quad+\sum_{j=1}^{k} C\left\{\left(\varepsilon 2^{j}\right)+\left(\varepsilon 2^{j}\right)^{\frac{1}{2}}+\left(2^{-j}\right)^{\rho}\right\}\left\{\left(f_{D_{2}}\left|u_{0}\right|^{2} d x\right)^{\frac{1}{2}}+\|F\|_{L^{p}\left(D_{2}\right)}+\|h\|_{W^{1, p}\left(D_{2}\right)}+\|\nabla g\|_{C^{0, \eta}\left(\Delta_{2}\right)}\right\} .
\end{aligned}
$$

Noting that $0<\varepsilon<2^{-k}$, there exists a constant $C$ independent of $k$ such that

$$
\begin{aligned}
\left|f_{D_{r}} p_{\varepsilon}-f_{D_{1}} p_{\varepsilon} d x\right| \leq C & \left\{\left(f_{D_{2}}\left|u_{\varepsilon}\right|^{2} d x\right)^{\frac{1}{2}}+\left(f_{D_{2}}\left|u_{0}\right|^{2} d x\right)^{\frac{1}{2}}+\left(f_{D_{2}}|F|^{p} d x\right)^{\frac{1}{p}}\right. \\
+ & \left.\left(f_{D_{2}}|\nabla h|^{p} d x\right)^{\frac{1}{p}}+\|h\|_{L^{\infty}\left(D_{2}\right)}+\|\nabla g\|_{C^{0, \eta}\left(\Delta_{2}\right)}\right\} .
\end{aligned}
$$


In fact, we know that $u_{\varepsilon} \rightarrow u_{0}$ strongly in $L^{2}\left(D_{2} ; \mathbb{R}^{d}\right)$ according to the construction of $\left(u_{0}, p_{0}\right)$ in Lemmas 4.3 and 5.3. Hence, it is not hard to see that

$$
\left(f_{D_{2}}\left|u_{0}\right|^{2} d x\right)^{\frac{1}{2}} \leq C\left(f_{D_{2}}\left|u_{\varepsilon}\right|^{2} d x\right)^{\frac{1}{2}}
$$

Put this inequality into (5.12), and we finally derive the desired estimate.

Proof of Theorem 1.2. The desired estimate (1.6) directly follows from Theorems 4.1 and 5.1.

Proof of Theorem 1.1. The estimate (1.5) follows from Theorem 1.2 and [13, Theorem 1.1], and we are done.

\section{Acknowledgements}

Both of the authors want to express their sincere appreciation to Professor Zhongwei Shen for his constant guidance and encouragement. The second author was supported by the National Natural Science Foundation of China (Grant NO.11471147).

\section{References}

[1] S. Armstrong, C. Smart, Quantitative stochastic homogenization of convex integral functionals, Ann. Sci. Éc. Norm. Supér., (to appear).

[2] S. Armstrong, Z. Shen, Lipschitz estimates in almost-periodic homogenization, Comm. Pure Appl. Math. 69(2016), no.10, 1882-1923.

[3] M. Avellaneda and F. Lin, Compactness methods in the theory of homogenization, Comm. Pure Appl. Math. 40(1987), no.6, 803-847.

[4] A. Bensoussan, J.-L. Lions, and G.C. Papanicolaou, Asympotic Analysis for Periodic Structures, Studies in Mathematics and its Applications, North Holland, 1978.

[5] M. Giaquinta, G. Modica, Non-linear systems of the type of the stationary Navier-Stokes system, J. Reine Angew. Math. 330(1982), 173-214.

[6] G.P. Galdi, C.G. Simader, Existence, uniqueness and $L^{q}$ estimates for the Stokes problem in an exterior domain, Arch. Rational Mech. Anal. 112(1990), 291-318.

[7] J. Geng, Z. Shen, Uniform regularity estimates in parabolic homogenization, Indiana Univ. Math. J. 64 (2015), no.3, 697-733.

[8] J. Geng, Z. Shen, Convergence rates in parabolic homogenization with time-dependent coefficients, J. Funct. Anal. (in press).

[9] J. Geng, Z. Shen, L. Song, Boundary Korn inequality and Neumann problems in homogenization of systems of elasticity, arXiv:1608.07736v1(2016).

[10] S. Gu, Convergence rates in homogenization of Stokes systems, J. Differential Equations 260(2016), no.7, 5796-5815. 
[11] S. Gu, Convergence Rates of Neumann problems for Stokes Systems, arXiv:1512.08285.

[12] S. Gu,Homogenization of Stokes Systems with Periodic Coefficients, Thesis (Ph.D.) University of Kentucky, 2016.

[13] S. Gu, Z. Shen, Homogenization of Stokes systems and uniform regularity estimates, SIAM J. Math. Anal. 47(2015), no.5, 4025-4057.

[14] C.E. Kenig, F. Lin, Z. Shen, Homogenization of elliptic systems with Neumann boundary conditions, J. Amer. Math. Soc. 26(2013), no.4, 901-937.

[15] C.E. Kenig, F. Lin, Z. Shen, Convergence rates in $L^{2}$ for elliptic homogenization problems, Arch. Ration. Mech. Anal. 203(2012), no.3, 1009-1036.

[16] O.A. Ladyzhenskaya, The Mathematical Theory of Viscous Incompressible Flow, Revised English edition, Gordon and Breach Science Publishers, New York-London, 1963.

[17] Z. Shen, Boundary estimates in elliptic homogenization, arXiv:1505.00694v1(2015).

[18] Z. Shen, J. Zhuge, Boundary layers in periodic homogenization of Neumann problems, arXiv:1610.05273v2(2016).

[19] Z. Shen, J. Zhuge, Approximate correctors and convergence rates in almost-periodic homogenization, arXiv:1603.03139v2(2016).

[20] T. Suslina, Homogenization of the Dirichlet problem for elliptic systems: $L_{2}$-operator error estimates, Mathematika 59(2013), no.2, 463-476.

[21] T. Suslina, Homogenization of the Neumann problem for elliptic systems with periodic coefficients, SIAM J. Math. Anal. 45(2013), no.6, 3453-3493.

[22] Q. Xu, Convergence reates and $W^{1, p}$ estimates in homogenization theory of Stokes systems in Lipschitz domains, arXiv:1609.00122v1 (2016).

[23] Q. Xu, Convergence rates for general elliptic homogenization problems in Lipschitz domains, SIAM J. Math. Anal. 48(2016), no.6, 3742-3788.

[24] Q. Xu, Uniform regularity estimates in homogenization theory of elliptic systems with lower order terms on the Neumann boundary problem, J. Differential Equations 261(2016), no.8, 4368-4423.

[25] V.V. Zhikov, S.E. Pastukhova, On operator estimates for some problems in homogenization theory, Russ. J. Math. Phys. 12(2005), no. 4, 515-524.

[26] J. Zhuge, Uniform boundary regularity in almost-periodic homogenization. J. Differential Equations 262(2017), no.1, 418-453. 